\title{
RACSAM
}

Rev. R. Acad. Cien. Serie A. Mat.

VOL. 102 (1), 2008, pp. 127,159

Geometría y Topología / Geometry and Topology

Artículo panorámico / Survey

\section{Dynamical systems and shapes}

\section{J. J. Sánchez-Gabites}

\begin{abstract}
This survey is an introduction to some of the methods, techniques and concepts from algebraic topology and related areas (homotopy theory, shape theory) which can be fruitfully applied to study problems concerning continuous dynamical systems. To this end two instances which exemplify the interaction between topology and dynamics are considered, namely, Conley's index theory and the study of some properties of certain attractors.
\end{abstract}

\section{Sistemas dinámicos y formas}

Resumen. Este artículo panorámico constituye una introducción a algunos de los métodos, técnicas y conceptos que, desde la topología algebraica y otras áreas afines (teoría de homotopía, teoría de la forma), permiten abordar problemas que se plantean en el marco de los sistemas dinámicos continuos. Para ello se presentan dos situaciones que ejemplifican esta interacción entre topología y dinámica, como son la construcción del índice de Conley y el estudio de algunas propiedades de ciertos atractores.

Generally speaking, the task of applied sciences is to observe natural phenomena and try to elaborate a theory which explains them. Such a theory is frequently formalized (at least in quantitative sciences) in a mathematical language and can be used to produce simple models of the phenomena, usually in the form of a dynamical system 1 . Then a mathematical analysis of the latter can be performed and may provide explanations for the basic features of the observed behaviour.

The study of dynamical systems involves many areas of mathematics, most notably analysis and topology. More specifically, algebraic topology entered the picture through the pioneering work of Poincaré, later continued by Morse, Smale and Conley, which showed that there exists a strong interaction between a dynamical system and the shape (in an informal, intuitive sense) of the phase space it lives in.

Their methods can be considered landmarks in the study of dynamical systems through their phase portraits, which are objects of a geometrical nature. This approach gave rise to a whole new branch where tools like homotopy theory, homology and cohomology theories, and later on shape theory, played a prominent role in the investigation of dynamical systems.

The aim of this survey is to present a (necessarily partial and strongly biased) illustration of two specific instances which exemplify how the tools mentioned above are brought into the picture of dynamical systems. We construct Conley's index in its shape theoretical version, present the subsequent Morse equations (this is Section 2] and explore some results about attractors (Section 3). The exposition is very unbalanced in the sense that a great space is taken up by Section 2 but this is just a natural consequence of the fact that

Presentado por José María Montesinos.

Recibido: 26 de junio de 2007. Aceptado: 5 de diciembre de 2007.

Palabras clave / Keywords: Topological dynamics, Conley index, shape theory.

Mathematics Subject Classifications: 54H20, 55P55.

(c) 2008 Real Academia de Ciencias, España.

${ }^{1}$ All the words in slanted letters will be defined later in the survey. 
it condenses nearly one hundred years of beautiful and deeply influential mathematics and provides enough background for an interested reader to explore more advanced topics. Unfortunately we will not be able even to mention the all important robustness feature of Conley's index.

Only a general background in topology and in particular in algebraic topology is needed (for the latter, the books [33], [70] and [71] contain more than enough information), although a mild acquaintance with the theory of (at least linear) differential equations is recommended (as presented in [31], for example). We have chosen to recall the relevant notions or results as they appear along the text. This has the advantage of presenting them in a motivated way, but on the other hand they can interrupt the discourse, so we introduce these digressions by a short title in boldface and end them by a horizontal line to allow the reader to recognize when he is back to the main story.

\section{Introduction}

Before proceding further we will recall some elementary concepts and fix notations. The basic reference here, which we shall follow closely, is the book by Bhatia and Szegö [8]. Besides its mathematical content, a quick glance at its introductory pages will give the reader a fairly accurate picture of the most prominent figures involved in the development of the theory of dynamical systems until the 70's, and this can provide a head start in the arduous task of untangling the many approaches which exist nowadays to this subject. Another two very complete resources, with a stronger bias towards phase portraits, are [52] and [53].

\subsection{Basic definitions}

Suppose we want to study the evolution in time of some physical system $(S)$ whose state can be completely described at any instant $t \in \mathbb{R}$ by means of a vector $S(t) \in D \subseteq \mathbb{R}^{n}$. Here $S(t)$ can be thought of as a "photograph" of $(S)$ at time $t$, and $D$ is the set of admissible states for $(S)$. Assuming that the system is well behaved (it is deterministic both in the future and in the past), knowledge of $S\left(t_{0}\right)$ at any particular instant $t=t_{0}$ completely determines $S(t)$ for every $t \in \mathbb{R}$. Hence given any $p \in D$ we can run $(S)$ using $p$ as its initial state (that is, letting $S(0)=p$ ) to obtain a curve

$$
\begin{aligned}
\gamma_{p}: \mathbb{R} & \longrightarrow D \\
t & \longmapsto S(t)
\end{aligned}
$$

representing the evolution of $(S)$ in time. Collecting all these together yields a new mapping

$$
\begin{aligned}
\varphi: D \times \mathbb{R} & \longrightarrow D \\
(p, t) & \longmapsto \gamma_{p}(t)
\end{aligned}
$$

which satisfies $\varphi(p, 0)=\gamma_{p}(0)=p$ for every $p \in D$. Furthermore, we shall assume that the system is autonomous, this meaning that the laws which govern it do not depend on time. We shall not explore this in detail, but it has the consequence that the evolutions

$$
\begin{aligned}
& p \stackrel{t+s}{\longrightarrow} \varphi(p, t+s) \\
& p \stackrel{t}{\longrightarrow} \varphi(p, t) \stackrel{s}{\longrightarrow} \varphi(\varphi(p, t), s)
\end{aligned}
$$

must yield the same final result, that is $\varphi(p, t+s)=\varphi(\varphi(p, t), s)$.

Definition 1 A continuous dynamical system (or flow) in a topological space $M$ (called the phase space) is a continuous mapping $\varphi: M \times \mathbb{R} \rightarrow M$ such that 
1. $\varphi(p, 0)=p \forall p \in M$,

2. $\varphi(\varphi(p, t), s)=\varphi(p, t+s) \forall p \in M, s, t \in \mathbb{R}$.

Although Definition 1 is probably well known (simply put, $\varphi$ is a continuous action of the additive group $(\mathbb{R},+)$ on the topological space $M)$, we have given some motivation for it to draw attention on the fact that one could as well consider other situations which exhibit interest on their own. Let us present two of them here:

- If $(S)$ is deterministic only in the future, knowledge of state $S\left(t_{0}\right)$ does not always determine the past history of $(S)$ (prototypical examples being heating or diffusion phenomena) and the curves $\gamma_{p}$ are defined only for $t \geq 0$. Therefore, it is natural to modify Definition 1 by requiring that the domain of $\varphi$ be only $M \times[0,+\infty)$, rather than $M \times \mathbb{R}$, and keeping axioms 1 and 2 from Definition 1 for $s$, $t \geq 0$. The notion thus obtained is called a continuous semidynamical system.

- $(S)$ could evolve in a discrete fashion (for example, it could change its state on a daily basis, instead of continuously with time), and then it would be reasonable to replace $\mathbb{R}$ by $\mathbb{Z}$ in Definition 1 These are known as discrete dynamical systems.

This survey concentrates on continuous dynamical systems because they are best suited to be studied with geometric techniques, since many of the constructions and results described below rely on the simple idea of using the flow to construct homotopies between adequate mappings. However, many of the forthcoming concepts do have their discrete or semidynamical counterparts which have also proved to be useful.

Let us fix some conventions. We shall always reserve the letter $\varphi$ for flows, and abbreviate, as customary, $\varphi(p, t)$ by $p \cdot t$. The phase space $M$ will be assumed to be locally compact and metrizable (in fact we will usually confine ourselves, for simplicity, to the case where $M$ is a topological manifold). We would like to remark here that the local compactness assumption prevents this techniques from working straightforward in infinite dimensional phase spaces, which may arise in settings related to partial differential equations or quantum mechanics. Nevertheless, in many cases this issue can be succesfully fixed (see [55]).

Definition 2 The trajectory of a point $p$ is the set $p \cdot \mathbb{R}$. The integral curve through $p$ is the parametrized curve $\gamma_{p}: \mathbb{R} \rightarrow M$ given by $\gamma_{p}(t):=\varphi(p, t)$ (thus $\gamma_{p}(0)=p$ ).

Definition 3 A set $K \subseteq M$ is called invariant if $K \cdot \mathbb{R} \subseteq K$, that is, if the trajectory of every $p \in K$ is completely contained in $K$.

Since trajectories are connected sets (being the image of $\mathbb{R}$ under a continuous mapping), every component of an invariant set is again invariant. Morever the interior, closure and boundary of an invariant set is again invariant. So are their unions and intersections. Finally, if $K$ is invariant, the restriction $\left.\varphi\right|_{K \times \mathbb{R}}: K \times \mathbb{R} \rightarrow K$ is well defined and trivially satisfies the conditions to be a flow in $K$, which we call the restriction flow $\left.\varphi\right|_{K}$.

Example 1 The trajectory $p \cdot \mathbb{R}$ of a point $p$ is an invariant set, since $(p \cdot \mathbb{R}) \cdot \mathbb{R}=p \cdot(\mathbb{R}+\mathbb{R})=p \cdot \mathbb{R}$. We shall single out two classical types of points whose behaviour has a strong dynamical significance:

1. A point $p \in M$ is a fixed point (or an equilibrium, or a critical point) if $p \cdot t=p$ for all $t \in \mathbb{R}$, that is, if $\{p\}$ is invariant. In this case the trajectory of $p$ is just the singleton $\{p\}$.

2. A point $p \in M$ is periodic of (minimal) period $T>0$ if $p \cdot T=p$ but $p \cdot t \neq p$ for every $0<t<T$. In this case $p \cdot t=p \cdot s \Leftrightarrow t \equiv s \bmod T$ and the trajectory of $p$ is homeomorphic to $\mathbb{S}^{1}$, the unit circumference.

Let $\varphi: M \times \mathbb{R} \rightarrow M$ and $\psi: N \times \mathbb{R} \rightarrow N$ be dynamical systems in possibly different phase spaces $M$ and $N$. As usual in mathematics, it will be convenient to have some notion of equivalence between $\varphi$ and $\psi$ which allows us to deem them as the same dynamical system. 
Definition 4 A homeomorphism $h: M \rightarrow N$ is a conjugation between $\varphi$ and $\psi$ if $\psi(h(p), t)=(h \circ$ $\varphi)(p, t)$ for every $p \in M$ and $t \in \mathbb{R}$. When such a homeomorphism exists, we shall shay that $\varphi$ and $\psi$ are conjugate.

Clearly conjugate systems are indistinguishable from the topological point of view. However this relation is still too strict for our geometrical approach, and we shall have occasion later on to slacken it a little.

Many questions concerning flows involve their long term behaviour or, intuitively speaking, the values $\varphi(p,+\infty)=p \cdot(+\infty)$ of the flow at infinity. We shall devote a few lines to recall how this notion is formalized under the name of limit sets.

Fix a point $p \in M$ in the phase space. We want to extend the continuous mapping $\gamma_{p}: \mathbb{R} \rightarrow M$ to the domain $\mathbb{R} \cup\{+\infty\}$, that is ascribe a value to $\gamma_{p}(+\infty)=p \cdot(+\infty)$. If this is to be done in a continuous fashion, taking any sequence $t_{n} \rightarrow+\infty$ we must have $\gamma_{p}\left(t_{n}\right)=p \cdot t_{n} \rightarrow \gamma_{p}(+\infty)=p \cdot(+\infty)$, which in principle determines $p \cdot(+\infty)$. However different choices of the sequence $t_{n}$ might give rise to different limits for $p \cdot t_{n}$, or even to sequences having no limit whatsoever, so we are led to think of $p \cdot(+\infty)$, rather than a single point, as the following set:

Definition 5 The $\omega$-limit se日 of a point $p \in M$ is

$$
\omega(p):=\left\{q \in M: \exists t_{n} \rightarrow+\infty \text { such that } p \cdot t_{n} \rightarrow q\right\} .
$$

The $\omega$-limits are invariant sets, since

$$
\omega(p) \cdot t=(p \cdot(+\infty)) \cdot t=p \cdot(+\infty+t)=p \cdot(+\infty)=\omega(p)
$$

(this "proof" should be taken as a shorthand for a longer one). It is not difficult to see either that $\omega(p)$ admits the alternative definition

$$
\omega(p)=\bigcap_{t \geq 0} \overline{p \cdot[t,+\infty)}
$$

which has the advantage of showing readily that $\omega(p)$ is closed. Also, it is prone to generalization, and for any subset $P \subseteq M$ we define its $\omega$-limit as

$$
\omega(P):=\bigcap_{t \geq 0} \overline{P \cdot[t,+\infty)}
$$

All the definitions above can be dualized to obtain the notion of the $\alpha$-limit set of a point $p$, which we denote by $\alpha(p)$. It has properties completely analogous to those of $\omega(p)$.

\subsection{Phase portraits}

Let us recall that a phase portrait of a dynamical system $\varphi: M \times \mathbb{R} \rightarrow M$ is an informal drawing (so to speak) of the phase space $M$ together with some oriented trajectories of the system which are of interest or thought to be representative of the behaviour of the flow. Besides their heuristic relevance, phase portraits provide the link which allows geometry to enter the scene of dynamical systems.

Proposition 1 Let $\varphi$ be a continuous flow in M. Then every two trajectories of $\varphi$ either coincide or are disjoint. In particular, the collection of all trajectories of $\varphi$ is a partition of $M$.

Proof. Assume that two trajectories $p \cdot \mathbb{R}$ and $q \cdot \mathbb{R}$ meet. Then there exist times $s, t \in \mathbb{R}$ such that $p \cdot s=q \cdot t$, so $p \cdot(s-t)=q \cdot(t-t)=q \cdot 0=q$. Hence $q \in p \cdot \mathbb{R}$ and consequently $q \cdot \mathbb{R} \subseteq p \cdot \mathbb{R}$ because the latter is invariant (see Example 1). The same argument, interchanging the roles of $p$ and $q$, proves the other inclusion.

\footnotetext{
${ }^{2}$ Let us warn the reader that we exceptionally depart from the notation in $\left[8\right.$, where the $\omega$-limits are denoted by $\Lambda^{+}$.
} 
For us, the phase portrait of $\varphi$ will be the particular partition of $M$ induced by the trajectories of $\varphi$, together with their orientation. It captures all the essential behaviour of the flow except for the specific parametrization of its integral curves and, roughly speaking, the geometric theory of dynamical systems proceeds by studying these phase portraits, rather than the original flows which give rise to them. This shift of our focus is best exemplified by the following notion of equivalence of dynamical systems, coarser than that of conjugation.

Definition 6 Let $\varphi: M \times \mathbb{R} \rightarrow M$ and $\psi: N \times \mathbb{R} \rightarrow N$ be two dynamical systems (probably defined in different phase spaces). A homeomorphism $h: M \rightarrow N$ is called an equivalence between $\varphi$ and $\psi$ if it takes oriented trajectories of the former onto oriented trajectories of the latter.

Hence two flows are equivalent when there is a homeomorphism $h: M \rightarrow N$ taking the phase portrait of one onto that of the other, and therefore they are indistinguishable for the geometric theory of dynamical systems. Sometimes we shall also need a local version of Definition 6 and say that $\varphi$ and $\psi$ are locally equivalent in sets $U \subseteq M$ and $V \subseteq N$ if there exists a homeomorphism $h: U \rightarrow V$ taking oriented trajectory segments in $U$ to oriented trajectory segments in $V$ (the trajectory segment of a point $p \in U$ is just the set $p \cdot J$, where $J$ is the connected component of $\{t \in \mathbb{R}: p \cdot t \in U\}$ which contains 0 ).

DYNAMICAL SYSTEMS COMING FROM DifFERENTIAL EQUATIONS. Many natural phenomena can be modelized by means of a differential equation which can be put (maybe after some manipulations) in the form

$$
(E): \dot{p}(t)=f(p(t))
$$

where $f$ is a tangent vector field on some manifold $M$. Under the assumption that $f$ be locally lipschitzian (for example, if it is of class $\mathcal{C}^{1}$ ), prescription of an initial condition $p(0)=q$ uniquely determines a maximal solution $p(t)$ for $(E)$. However, the domain of $p(t)$ does not need to be the whole real line $\mathbb{R}$, but only an open interval $J_{q}$ which may depend on the initial condition $q$. One can still collect all the solutions to obtain a local flow $\varphi: W \rightarrow M$, where $W=\bigcup_{q \in M}\{q\} \times J_{q}$ is an open subset of $M \times \mathbb{R}$ containing $M \times\{0\}$ and $\varphi$ satisfies axioms 1 and in Definition 1 wherever it makes sense (proofs of this facts are standard, see for example [13] or [31]). Despite appearances, we can also deal with local flows in our setting, since as far as phase portraits are concerned, these behave exactly like global flows (those of Definition 11. More precisely, one has the following result:

Proposition 2 Let $\varphi$ be a local flow on $M$. Then there exists a (global) flow $\widehat{\varphi}$ in $M$ such that the oriented trajectories of $\varphi$ and $\widehat{\varphi}$ coincide. Consequently their phase portraits are the same.

We shall not give its proof, but it follows rather easily from a version of Theorem 1 below for local flows and Whitney's paper [73], where sufficient conditions are given for a family of curves in a space $M$ to be the integral curves of a global flow. It can also be instructive to see how the book by Bhatia and Szegö deals with this issue in the particular case of local flows coming from differential equations, see [8] Chapter I, 2.2.]. In any case, whenever a flow defined by a differential equation is considered in the sequel, no precaution about its domain of definition will be necessary.

Concerning the aspect of phase portraits, there are a pair of results which the reader should be aware of. The first one (whose proof can be found, for example, in [8], or in [52]) shows that, away from fixed points, phase portraits are locally trivial.

Theorem 1 (Rectification theorem) If $p$ is not an equilibrium for $\varphi$, it possesses an open neighbourhood (called a flowbox) where the flow is parallel, that is, it resembles Figure 1

The second one concerns the topology of trajectories.

Theorem 2 The trajectories of $\varphi$ can be classified according to Table 1

The proof is not difficult except for the last case, considered in a paper by Aarts [1]. 


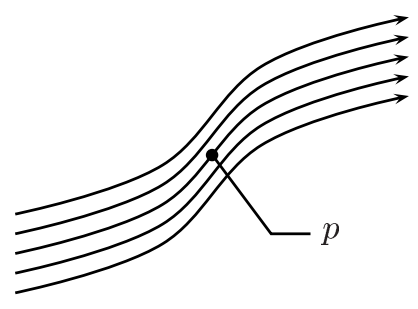

Figure 1. A parallel flow.

Table 1. Dynamics versus topology of trajectories

\begin{tabular}{|c|c|c|}
\hline Dynamics of $p$ & Type of $p \cdot \mathbb{R}$ & Topology of $p \cdot \mathbb{R}$ \\
\hline \hline Critical & $\{p\}$ & A singleton \\
\hline Periodic & $\mathbb{S}^{1}$ & Compact but not a singleton \\
\hline Neither critical nor periodic & $\mathbb{R}$ & Locally compact but not compact \\
\cline { 2 - 3 } & Locally $\cong \mathbb{Q} \times \mathbb{R}$ & Not locally compact \\
\hline
\end{tabular}

Example 2 Suppose a continuous flow is given in the unit circumference $\mathbb{S}^{1}$ which has no fixed points. Then every point $p \in \mathbb{S}^{1}$ is periodic, and its orbit is the whole phase space $\mathbb{S}^{1}$.

PROOF. Observe first that $p \cdot \mathbb{R}$ is a connected subset of $\mathbb{S}^{1}$, so it is an arc (either proper or the whole circumference). In any case it is locally compact, so by Table 1 it follows (since there do not exist fixed points) that $p$ is either periodic or $p \cdot \mathbb{R}$ is homeomorphic to $\mathbb{R}$. If the latter were the case, then $p \cdot \mathbb{R}$ would be an open oriented arc $\left(\theta_{1}, \theta_{2}\right)$ and $\omega(p)=\left\{\theta_{2}\right\}$ would be an invariant singleton, thus a fixed point. This contradicts the assumption that there are no fixed points, so $p$ must be periodic and $p \cdot \mathbb{R}=\mathbb{S}^{1}$.

To close this section we present an example which is probably well known by the reader, but looked upon with geometrical techniques. Despite its simplicty we hope it will transmit some of the flavour of our subject.

Example 3 Consider a system comprised of a spring with one of its ends fixed and the other attached to a mass $m$. The spring offers a force $g(x)$ against being displaced from its rest position by an amount $x$. That $i$, if the natural length of the spring is $L$ and it is pulled apart so that it attains length $L+x$, it will show a force $g(x)$ pulling back to its rest position, and similarly if shortened to length $L-x$ (see Figure 2).

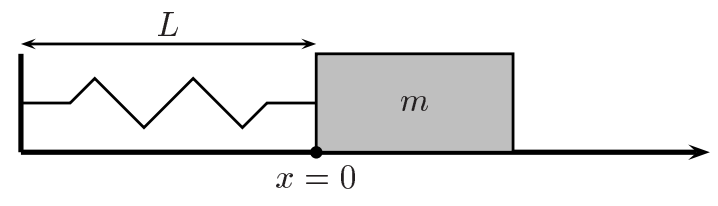

Figure 2. The set up for Example 3 .

We claim that if $g$ is continuous in some interval $(\alpha, \beta) \subseteq \mathbb{R}$ containing $x=0$, then the spring exhibits periodic behaviour when its rest position is slightly perturbed.

PROOF. We adopt the reference system of Figure 2 Applying Newton's second law to the above situation (and neglecting friction), the position $x(t)$ of the particle $m$ is subject to the differential equation $m \ddot{x}(t)=$ $-g(x(t))$ which, under the standard transformation to a first order system, yields

$$
(S):\left\{\begin{array}{ccc}
\dot{x} & = & y \\
m \dot{y} & = & -g(x)
\end{array}\right.
$$


Since the effect of friction has been neglected, system $(S)$ is conservative: the total energy

$$
V(x, y):=G(x)+\frac{m}{2} y^{2}
$$

is a conserved quantity, where $G(x):=\int_{0}^{x} g(u)$ du. Indeed, if $p(t)=(x(t), y(t))$ is a solution of $(S)$,

$$
(V \circ p)^{\prime}=\nabla_{p} V \cdot p=\left(G^{\prime}(x), m y\right)\left(\begin{array}{c}
y \\
\frac{-1}{m} g(x)
\end{array}\right)=0
$$

because $G^{\prime}=g$. Hence the level sets $V^{-1}(c)$ are invariant for every $c \in \mathbb{R}$. Moreover, the implicit function theorem guarantees that $V^{-1}(c)$ is a closed 1-manifold (without boundary) for every $c>0$, since $\nabla V(x, y)=(g(x), m y)$ does not vanish except at $(x, y)=(0,0)$, which corresponds to $V=0$.

Observe that $g(x)$ opposes the external force, so we have $g(x)>0$ for $x>0, g(0)=0$ (rest position) and $g(x)<0$ when $x<0$. Therefore $G$, being a primitive of $g$, is strictly decreasing for negative $x$, attains its minimum at $x=0$ and then increases strictly again for positive $x$. Thus for small enough $c>0$ the set $\{x \in(\alpha, \beta): G(x) \leq c\}$ is a closed interval $\left[x_{1}, x_{2}\right]$. Furthermore, $G \geq 0$ so if $(x, y) \in V^{-1}(c)$, then

$$
c=V(x, y)=G(x)+\frac{m}{2} y^{2} \geq \frac{m}{2} y^{2} \Rightarrow y \in\left[-\sqrt{\frac{2 c}{m}}, \sqrt{\frac{2 c}{m}}\right]
$$

and consequently

$$
V^{-1}(c) \subseteq\left[x_{1}, x_{2}\right] \times\left[-\sqrt{\frac{2 c}{m}}, \sqrt{\frac{2 c}{m}}\right],
$$

which shows that $V^{-1}(c)$ is bounded and therefore compact. Thus if we denote by $K$ any of its connected components, $K$ is a circumference $\mathbb{S}^{1}$ (being a connected, compact 1-manifold). Furthermore, $K$ is invariant (because it is a component of an invariant set) and does not contain any critical points, since only the origin is a critical point and $V(0,0)=0<c=V(K)$. Thus by Example 2 it is a periodic orbit.

\section{Local and global analysis of dynamics}

One approach to dynamical systems, inaugurated by Poincaré in his series of papers [58] and continued by Morse [49], [50], Smale [67], [68] and Conley [14], [16], could be roughly described as follows. First of all, a finite family of invariant sets $K_{1}, \ldots, K_{m} \subseteq M$ is located such that the flow in $M-\bigcup_{j=1}^{m} K_{j}$ is especially simple. Then some kind of local analysis is performed around those sets (the term "local" meaning that it involves only the flow in arbitrarily small neighbourhoods of the invariant sets), whose outcome is an index (whatever that may be) which is assigned to each of $K_{1}, \ldots, K_{m}$. Finally, this local information is put together in some way to fit in a large picture involving some global topology of the phase space, for example its Betti numbers.

I. PoINCARÉ. Poincaré (around 1880) dealt with continuous flows in compact surfaces $M$ with finitely many fixed points $\left\{p_{1}, \ldots, p_{m}\right\}$, which play the role of the invariant sets $K_{1}, \ldots, K_{m}$ (in this case no specific assumption is made about the flow in $M-\left\{p_{1}, \ldots, p_{m}\right\}$, which accounts for the generality of the resulting theory). He assigned an integer $\iota\left(p_{j}\right)$ to each of them, its Poincaré-Hopf index, and went on to prove the celebrated

Theorem 3 (Poincaré-Hopf for surfaces) Let $\varphi$ be a continuous flow in a closed surface $M$ with finitely many fixed points $p_{1}, \ldots, p_{m}$. Then

$$
\sum_{j=1}^{m} \iota\left(p_{j}\right)=\chi(M)
$$


Theorem 3 relates the local information provided by the indices with a global feature of the phase space, namely its Euler characteristic. This theory was later extended to flows in higher dimensions by Hopf [35], although reading Poincaré's original paper is still enjoyable due to its strong geometric flavour. Modern expositions of the topic can be found nearly in any book about differential topology, for example in [34] or [46].

II. MoRSE. Although having a completely different departure point, the work of Morse proved to be of crucial importance for the developments to come later on in the theory of dynamical systems. He realized that, if $f: M \rightarrow \mathbb{R}$ is a differentiable function (with some mild restrictions) defined on a compact riemannian manifold $M$, then the critical points $p_{1}, \ldots, p_{m}$ of $f$ (that is, those where the gradient $\nabla f$ vanishes) bore some relation to the topology of $M$. More concretely, he assigned an integral index to each critical point $p_{j}$ (comprised between 0 and $\operatorname{dim}(M)$, the dimension of $M$ ) which could be computed from the hessian of $f$ at $p_{j}$ (thus being a local object) and went on to prove some inequalities, now known as Morse's inequalities. These relate the amount of critical points having a certain index $k$ and the Betti numbers of $M$.

To make this fit our context, one can consider the differential equation $(E): \dot{p}(t)=-\nabla_{p(t)} f$ (the minus sign is included here for historical reasons), which induces a global flow $\varphi_{f}$ in $M$ (due to its compactness) whose fixed points are precisely the critical points $p_{1}, \ldots, p_{m}$ of $f$. With an adequate choice of their labeling one can prove (see Example 4 and Proposition 3] that, if $p \neq p_{1}, \ldots, p_{m}$,

$$
(P): \alpha(p)=p_{s} \text { and } \omega(p)=p_{r} \text { for some equilibria } p_{s} \text { and } p_{r} \text { such that } m \geq s>r \geq 1 .
$$

The fixed points play the role of the invariant sets $K_{1}, \ldots, K_{m}$ and the "niceness" assumption on the flow on $M-\bigcup_{j=1}^{m} K_{j}$ is precisely the above mentioned property $(P)$ that the trajectories of non critical points connect two equilibria. The local information provided by the indices of the critical points is put together via Morse's inequalities.

III. Smale. Nearly thirty years later, Smale considered differentiable flows in compact manifolds $M$ with finitely many fixed points $\left\{p_{1}, \ldots, p_{m}\right\}$ and periodic orbits $\left\{\gamma_{1}, \ldots, \gamma_{n}\right\}$ (these are the invariant sets $K_{j}$; the flow in their complement is assumed to satisfy a straightforward generalization of property $(P)$ above and some extra transversality condition which we shall not detail). An integral numerical index was assigned to each of them and inequalities relating the number of fixed points and periodic orbits with the Betti numbers of $M$, much in the fashion of Morse's, were obtained. Thus Smale, apart from generalizing Morse's work, took the important step of placing it in the context of dynamical systems.

IV. ConLeY. It was finally Conley who dealt with the case of nearly completely general invariant sets $K_{1}, \ldots, K_{m}$ (he required them to be isolated) in a compact space $M$. His index $h\left(K_{j}\right)$ (the Conley index) is a homotopy type of a pointed space, rather than a numerical index, and a theorem is obtained which relates those indices with the Betti numbers of $M$ whenever $\left\{K_{1}, \ldots, K_{m}\right\}$ is a Morse decomposition of $M$.

The reader might be wondering to what extent the approach presented above is general enough 1 One of the many merits of Conley's work is that he showed that the path Poincare had started to pave back in the 1880's effectively led somewhere (his ideas about this can be found in the monography [14], although they were already contained in a previous unpublished paper). We shall discuss this now, and to this end it is most convenient to introduce the following notion:

Definition 7 Let $\mathcal{K}=\left\{K_{j}\right\}_{j \in J}$ be a family of disjoint compact invariant subsets of $M$. A Lyapunov function for $\mathcal{K}$ is a continuous function $G: M \rightarrow \mathbb{R}$ such that:

\footnotetext{
${ }^{3}$ At least the dichotomy between local and global analysis arises naturally since, for instance, it was already present in Example 3 if not very prominently. Indeed, the usage of $V$ to locate $K$ involved the globality of the flow (though the topology of the phase space did not play an essential role here, apart from being 2-dimensional), whereas the conclusion that $K$ is a periodic orbit was drawn locally, from the knowledge that it did not contain fixed points.
} 
1. $G$ is strictly decreasing on the trajectories of points $p \notin \bigcup_{j \in J} K_{j}$,

2. G assumes a constant value $c_{j}$ on each $K_{j} \in \mathcal{K}$, and all of them are different.

The real numbers $c_{j}$ are called the critical values of $G$.

It is not easy to motivate Definition 7 without descending into technical details, and we shall not try to do so. But, if only to give a slight clue of where it comes from, let us present a prototypical example:

Example 4 Let $f: M \rightarrow \mathbb{R}$ be a differentiable function defined on compact manifold endowed with a riemannian metric $\langle\cdot, \cdot\rangle$ (recall that this is Morse's setting). Integration of the equation $\dot{p}(t)=-\nabla_{p(t)} f$ yields a flow $\varphi_{f}$ on $M$ whose fixed points are precisely the critical points of $f$. If $p$ is not one of them,

$$
\left.\frac{\mathrm{d}}{\mathrm{d} t}\left(f \circ \gamma_{p}\right)\right|_{t=0}=\left\langle\nabla_{p} f, \dot{\gamma}(p)\right\rangle=-\left\langle\nabla_{p} f, \nabla_{p} f\right\rangle<0
$$

so that $f$ is strictly decreasing along the trajectory of $p$. Thus letting $\mathcal{K}$ be the family of critical points of $f$, the function $f$ is a Lyapunov function for $\mathcal{K}$.

A careful examination of the work of Morse, when considered from the point of view of dynamical systems, shows that the fact that $f$ is a Lyapunov function was the key which enabled him to put together the local information provided by the indices and relate it with the Betti numbers of the ambient manifold. This may provide an (admittedly, not very satisfactory) explanation of why it is interesting to single out the notion of a Lyapunov function. Further justification comes from the Proposition 3

Proposition 3 Suppose that $\mathcal{K}=\left\{K_{j}\right\}_{j \in J}$ admits a Lyapunov function and $K:=\bigcup_{j \in J} K_{j}$ is compact. Then:

1. for every $p \in M$ there exist $K_{s}$ and $K_{r}$ in $\mathcal{K}$ such that $\alpha(p) \subseteq K_{s}$ and $\omega(p) \subseteq K_{r}$,

2. if $p \notin K$, then $\alpha(p)$ and $\omega(p)$ are nonempty and $\left.G\right|_{\alpha(p)}>G(p)>\left.G\right|_{\omega(p)}$.

In the second case, $p \cdot \mathbb{R}$ is called a connecting orbit from $K_{s}$ to $K_{r}$.

Proof. In the first place we shall prove that $\omega(p) \subseteq K$ for every $p \in M$. If not, there exists $p \in M$ such that $\omega(p) \nsubseteq K$; let $q \in \omega(p)-K$. There exists a sequence $t_{n} \rightarrow+\infty$ such that $p \cdot t_{n} \rightarrow q$ and, maybe after passing to a subsequence, we can assume that $t_{n+1}-t_{n}>1$ for every $n \in \mathbb{N}$. Since $K$ is closed and $q \notin K$, it follows that $p \cdot t_{n} \notin K$ for big enough $n$ so

$$
G\left(p \cdot t_{n}\right)<G\left(p \cdot\left(t_{n}+1\right)\right)<G\left(p \cdot t_{n+1}\right)
$$

and passing to the limit and using the continuity of $G$ we get that $G(q) \leq G(q \cdot 1) \leq G(q)$. Hence $G(q)=G(q \cdot 1)$, but this contradicts the fact that $G$ decreases on trajectories of points in $M-K$ and proves that $\omega(p) \subseteq K$.

Let us observe now that $\omega(p)$ is connected. It is compact, because it is a closed subset of $K$. Moreover, since $\omega(p)=\bigcap_{t \geq 0} \overline{p \cdot[t,+\infty)} \subseteq K$ and $M$ is locally compact, there must exist some $t_{0}>0$ such that $\overline{p \cdot[t,+\infty)}$ is compact for every $t \geq t_{0}$. Therefore $\omega(p)$ is the intersection of the decreasing sequence of compact sets $\overline{p \cdot[t,+\infty)}$ (for $t \geq t_{0}$ ), each of which is connected (being the closure of the connected set $p \cdot[t,+\infty)$ ), so $\omega(p)$ is connected too (the same argument also proves, in passing, that $w(p) \neq \emptyset$ ). Recalling that $\omega(p) \subseteq K=\bigcup_{j \in J} K_{j}$ and every $K_{j}$ is closed, $\omega(p)$ must be wholly contained in some $K_{r}$.

Now we prove the inequality $G(p)>\left.G\right|_{\omega(p)}$ for $p \notin K$. Pick any $q \in \omega(p)$ and let $t_{n} \rightarrow+\infty$ be such that $p \cdot t_{n} \rightarrow q$. We may as well suppose that $t_{n} \geq 1$ for every $n$, and then we have $G(p)>G(p \cdot 1)>$ $G\left(p \cdot t_{n}\right)$, which on the limit gives $G(p)>G(p \cdot 1) \geq G(q)$. Since $G$ is constant on $\omega(p)$ because the latter is contained in some member of the family $\mathcal{K}$, it follows that $G(p)>\left.G\right|_{\omega(p)}$.

Finally, parallel arguments establish the dual afirmations for $\alpha(p)$. 
Thus the dynamics in the $K_{j}$ 's may be unknown, but every point $p \notin \bigcup_{j \in J} K_{j}$ yields a connecting trajectory $p \cdot \mathbb{R}$ between two of them. This in an exact analogue of property $(P)$ mentioned above, when discussing the work of Morse, but where more general invariant sets are allowed instead of only fixed points. The situation we have arrived at is close (save for the fact that we would like to have only finitely many $K_{j}$ 's) to the one we presented intuitively at the beginning of this section. To round off these considerations let us cite the following theorem due to Conley (see [14 Chapter II, 6.4]).

Theorem 4 Let the phase space $M$ be compact. Then there exists a unique family $\mathcal{K}=\left\{K_{j}\right\}_{j \in J}$ of disjoint compact invariant sets which admits a Lyapunov function $G$ and is maximal with respect to this property. The set $K:=\bigcup_{j \in J} K_{j}$, called the chain recurrent set, is compact and the $K_{j}$ 's are its components.

Theorem 4 pushes things to the limit. It proves the existence of a family $\mathcal{K}$ which admits a Lyapunov function but cannot be further refined, that is, it separates completely the gradient-like part of the flow (that on which $G$ decreases) from the possibly complicated invariant structure. This can be considered as the ultimate justification to this approach to dynamical systems.

As a final outline let us recall the three steps we have to address and present the tools involved:

1. Locate invariant structure. This will be done via Ważewski’s method.

2. Analyze the flow near an invariant set $K$. The outcome is Conley's index $h(K)$, or rather a shapetheoretical version $s(K)$ of it.

3. Piece together the local information to obtain global constraints. This is accomplished through the Morse equations.

\subsection{Ważewski's method}

In his paper [72], Tadeusz Ważewski presented what was, by then, a new method to detect invariant structure in a prescribed compact subset $N \subseteq M$. Denote by $\operatorname{inv}(N)$ the maximal invariant subset of $N$, that is

$$
\operatorname{inv}(N):=\{p \in N: p \cdot \mathbb{R} \subseteq N\}
$$

Clearly $N$ contains a nonempty invariant subset if, and only if, $\operatorname{inv}(N) \neq \emptyset$.

Intuitively speaking, Ważewski's method proceeds by comparing how much of the "matter" in $N$ exits it when time goes by. Let us begin with a very simple example, the extreme case when nothing exits $N$.

Example 5 Suppose that every point $p \in N$ remains in $N$ in forward time, that is $N \cdot[0,+\infty) \subseteq N$ (such a set is called positively invariant). Then we assert that $\operatorname{inv}(N) \neq \emptyset$.

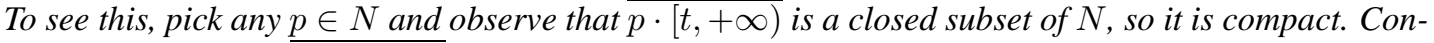
sequently $\omega(p)=\bigcap_{t>0} \overline{p \cdot[t,+\infty)}$ is an intersection of decreasing (with increasing $t$ ) nonempty compact sets, so it is nonempty. But since $\omega(p)$ is invariant, $\emptyset \neq \omega(p) \subseteq \operatorname{inv}(N)$. Thus $N$ contains some invariant structure.

Using similar arguments it is not difficult to check that $\operatorname{inv}(N)=\omega(N)$.

Observe that the compactness of $N$ played an essential role in Example 5 If this condition on $N$ were omitted, it could very well happen that the flow lines just "dissipated" over infinity, without giving birth to any invariant structure in $N$ (consider a translation flow $\varphi(x, t)=x+t$ in the real line and $N=\mathbb{R}$ ).

For later reference, and to aid intuition, let us keep in mind the next Figure 3 It shows four possible ways for the trajectory of a point $p \in \partial N$ to meet $N$ (it is not an exhaustive classification, but only representative enough). The boundary $\partial N$ is the thick horizontal line, and the gray part is the interior of $N$. In cases $(a)$ and $(b)$ we shall say that $p$ is a transverse exit (respectively entrance) point. In cases $(c)$ and $(d)$ we talk about an exterior (respectively interior) tangency. 

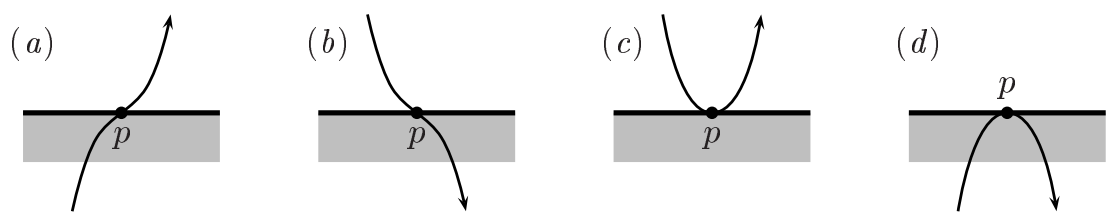

Figure 3. Possible phase portraits near a point $p \in \partial N$.

The usage of the words "transverse" or "tangent" here does not imply any differentiability assumptions, and in fact the cases depicted in Figure 3 can be completely characterized topologically. For example, $p$ is a transverse exit point if, and only if, there exists some $\varepsilon>0$ such that $p \cdot(-\varepsilon, 0) \subseteq \operatorname{int}(N)$ and $p \cdot(0, \varepsilon) \subseteq M-N$. Similarly, $p$ is an exterior tangency if, and only if, there exists some $\varepsilon>0$ such that $p \cdot(-\varepsilon, 0) \subseteq M-N$ and $p \cdot(0, \varepsilon) \subseteq M-N$.

Now to be able to state Ważewski's principle we need to make the following definition:

Definition 8 A point $p \in N$ is an (inmediate) exit point if, for every $\varepsilon>0$, we have $p \cdot[0, \varepsilon] \nsubseteq N$. The set of all exit points of $N$ will be denoted by $L$.

Thus in Figure 3 both in cases $(a)$ and $(c)$ the point $p$ is an exit point.

Observe that any point $p \in N$ which exits $N$ (that is, $p \cdot[0,+\infty) \nsubseteq \subseteq N$ ) does so through $L$. Indeed, let

$$
\tau(p):=\sup \{t \geq 0: p \cdot[0, t] \subseteq N\},
$$

which is finite because $p \cdot[0,+\infty) \nsubseteq N$. We claim that $p \cdot \tau(p) \in L$. If not, for some $\varepsilon>0$ we would have

$$
(p \cdot \tau(p)) \cdot[0, \varepsilon] \subseteq N,
$$

or equivalently $p \cdot[\tau(p), \tau(p)+\varepsilon] \subseteq N$. However, since $p \cdot[0, \tau(p)] \subseteq N$ because $N$ is closed and $\varphi$ is continuous, it would follow that

$$
p \cdot[0, \tau(p)+\varepsilon]=p \cdot[0, \tau(p)] \bigcup p \cdot[\tau(p), \tau(p)+\varepsilon] \subseteq N,
$$

contradicting the definition of $\tau(p)$. Hence $p \cdot \tau(p) \in L$.

Theorem 5 Assume $\operatorname{inv}(N)=\emptyset$. If $L$ is closed in $N$, then $L$ is a deformation retraction of $N$.

Proof. By the argument of Example 5 every $p \in N$ must exit $N$, because if some $p \in N$ did not, $\emptyset \neq \omega(p) \subseteq \operatorname{inv}(N)$. Thus the mapping $\tau$ described above is defined on all of $N$. The hypothesis that $L$ is closed guarantees that $\tau$ is continuous (we leave this as an exercise) and, since $N$ is compact, there exists some constant $T \geq 0$ such that $0 \leq \tau(p) \leq T$ for every $p \in N$. Consider the mapping

$$
\begin{aligned}
H: N \times[0, T] & \longrightarrow N \\
(p, t) & \longmapsto p \cdot \min \{t, \tau(p)\}
\end{aligned}
$$

The effect of $H$ on a point $p \in N$ as $t$ moves from 0 to $T$ is to push it along its trajectory until it reaches the exit set $L$, and thereafter leave it fixed. Clearly $H$ is continuous and, since $\tau(p) \leq T$, we have $H(p, T)=$ $p \cdot \tau(p) \in L$ for every $p \in N$. Thus $H$ is a deformation retraction of $N$ onto $L$.

Of course it is the following Corollary the result we are really interested in:

Corollary 1 Let $N$ be a compact set and suppose that the inmediate exit set $L$ is closed in $N$. If the inclusion $j: L \rightarrow N$ is not a homotopy equivalence, then $\operatorname{inv}(N) \neq \emptyset$. 
Proof. If $\operatorname{inv}(N)=\emptyset$, let $H$ be the deformation retraction provided by Theorem 5 If we let $r: N \rightarrow L$ be defined by $r(p):=H(p, T)$ we obtain a retraction of $N$ onto $L$, and $H$ provides a homotopy between the identity id $\operatorname{id}_{N}=H(\cdot, 0)$ and $r$. Hence $r$ is a homotopy inverse for the inclusion $j: L \rightarrow N$ and the latter is a homotopy equivalence, which contradicts the hypothesis.

Corollary 1 and Theorem [5] show how a dynamical question (whether there exists invariant structure in $N$ ) can be answered by means of a homotopy-theoretical criterion and introduce a technique which is prototypical in the geometric theory of dynamical systems, namely using the flow to construct homotopies. In fact Corollary 1 can be slightly reinforced by observing that the homotopy $H$ constructed in Theorem 5 leaves every point $p \in L$ fixed because $\tau(p)=0$ for them, so it induces a continuous mapping in the quotient $N / L$ which provides a strong deformation retraction of $N / L$ onto $L / L$. Thus

Corollary 2 Let $N$ be a compact set and suppose that the inmediate exit set $L$ is closed in $N$. If the pair $(N / L, L / L)$ does not have the homotopy type of the trivial pair $(*, *)$, then $\operatorname{inv}(N) \neq \emptyset$.

COMPUTING THE EXIT SET. Ważewski's method requires the computation of the exit set $L$. We will devote a few lines to show how this can be done in the frequent case when $N$ is a manifold with boundary and the flow is defined by a differential equation

$$
(E): \dot{p}(t)=f(p(t))
$$

where $f$ is of class $\mathcal{C}^{\infty}$. For simplicity the phase space $M$ will be assumed to be $\mathbb{R}^{n}$, but the argument carries over with minor changes to any other differentiable manifold.

First observe that $L \subseteq \partial N$. Indeed, if $p_{0}$ belongs to $\operatorname{int}(N)$, the interior of $N$ (which is an open set), the fact that $p_{0} \cdot 0=p_{0} \in \operatorname{int}(N)$ and the continuity of the flow imply that $p_{0} \cdot[0, t] \subseteq \operatorname{int}(N)$ for sufficiently small $t>0$ so $p_{0}$ does not belong to the exit set. Thus we only need a criterion to decide whether a given point $p_{0} \in \partial N$ belongs to $L$ or not.

Since $N$ is a manifold with boundary, it is defined (at least in some neighbourhood of $p_{0}$ ) by the inequality $h(p) \leq C$, where $h$ is a smooth function. It is a well known fact from differential geometry that $\nabla_{p_{0}} h$ is normal to $\partial N$ at $p_{0}$ and points in the direction of faster growth of $h$, which in our case is outwards of $N$. Hence if $\nabla_{p_{0}} h \cdot f\left(p_{0}\right)>0$, the vector $f\left(p_{0}\right)$ points outwards of $N$ too and consequently $\gamma_{p_{0}}$ exits $N$ through $p_{0}$. Reciprocally, when $\nabla_{p_{0}} \cdot f\left(p_{0}\right)<0$ the curve $\gamma_{p_{0}}$ enters $N$ through $p_{0}$. If the product is zero then $\gamma_{p_{0}}$ is tangent to $N$ at $p_{0}$ and we have to determine what the nature of the tangency is.

Let us present this in a formal way. Denoting by $\gamma_{p_{0}}(t)$ the integral curve through $p_{0}$ for $(E)$, we want to study the behaviour of $h$ along $\gamma_{p_{0}}(t)$, when $t$ approaches zero. Begin by expanding $\gamma_{p_{0}}$ around $t=0$ up to second order, obtaining

$$
\gamma_{p_{0}}(t)=\gamma_{p_{0}}(0)+\dot{\gamma}_{p_{0}}(0) \cdot t+\ddot{\gamma}_{p_{0}}(0) \cdot t^{2}+o\left(t^{2}\right) .
$$

Since $\gamma_{p_{0}}$ satisfies $(E)$ with initial condition $\gamma_{p_{0}}(0)=p_{0}$, it follows that $\dot{\gamma}_{p_{0}}(0)=f\left(\gamma_{p_{0}}(0)\right)=f\left(p_{0}\right)$ and, differentiating once more in $(E), \ddot{\gamma}_{p_{0}}(0)=D_{p_{0}} f \cdot f\left(p_{0}\right)$. Hence

$$
\gamma_{p_{0}}(t)=p_{0}+f\left(p_{0}\right) \cdot t+D_{p_{0}} f \cdot f\left(p_{0}\right) \cdot t^{2}+o\left(t^{2}\right) .
$$

Now expand $h$ around $p_{0}$ thus

$$
h(p)=C+\nabla_{p_{0}} h \cdot\left(p-p_{0}\right)+\frac{1}{2}\left(p-p_{0}\right)^{T} \cdot H_{p_{0}} h \cdot\left(p-p_{0}\right)+o\left(\left\|p-p_{0}\right\|^{2}\right),
$$

where $H_{p_{0}} h$ denotes the Hessian matrix of $h$ at $p_{0}$ and the superscript ${ }^{T}$ means transposition (we adopt the convention that vectors are columns). Plugging the expression for $\gamma_{p_{0}}(t)$ in place of $p$ and collecting terms we get

$$
\left(h \circ \gamma_{p_{0}}\right)(t)=C+\nabla_{p_{0}} h \cdot f\left(p_{0}\right) \cdot t+\left(\nabla_{p_{0}} h \cdot D_{p_{0}} f \cdot f\left(p_{0}\right)+\frac{1}{2} f\left(p_{0}\right)^{T} \cdot H_{p_{0}} h \cdot f\left(p_{0}\right)\right) \cdot t^{2}+o\left(t^{2}\right) .
$$

This gives inmediately the following criterion: 
Proposition 4 Let $p_{0} \in \partial N$ and assume that $N$ is defined by $h(p) \leq C$ near $p_{0}$.

1. If $\ell_{1}:=\nabla_{p_{0}} h \cdot f\left(p_{0}\right) \neq 0$, then $p_{0} \in L \Leftrightarrow \ell_{1}>0$.

2. If $\ell_{1}=0$ but $\ell_{2}:=\nabla_{p_{0}} h \cdot D_{p_{0}} f \cdot f\left(p_{0}\right)+\frac{1}{2} f\left(x_{0}\right)^{T} \cdot H_{p_{0}} h \cdot f\left(p_{0}\right) \neq 0$, then $p_{0} \in L \Leftrightarrow \ell_{2}>0$.

PRoof. Observe that $p_{0}$ is an exit point if, and only if, $g(t):=\left(h \circ \gamma_{p_{0}}\right)(t)>C$ for $t>0$ and sufficiently small. When $\ell_{1} \neq 0$, the first order term dominates in the expansion of $g(t)$ obtained above, so $g(t) \simeq$ $C+\ell_{1} t$ and $g(t)>C$ for $t>0$ if, and only if, $\ell_{1}>0$. When $\ell_{1}=0$ but the second order term $\ell_{2}$ does not vanish, $g(t) \simeq C+\ell_{2} t^{2}$ resembles a parabola so $g(t)>C$ for $t>0$ (in fact, for $t \neq 0$ ) if, and only if, $\ell_{2}>0$.

Higher order criteria can be obtained similarly, but we shall not need them.

Example 6 Consider the system of differential equations, in $\mathbb{R}^{3}$, given by

$$
(S)\left\{\begin{array}{l}
\dot{x}=\left(x-y \cos \frac{x}{2}\right)\left(x^{2}+y^{2}\right)-x \\
\dot{y}=(x+y)\left(x^{2}+y^{2}\right)-y \\
\dot{z}=-z
\end{array}\right.
$$

Since the right hand side $f(x, y, z)$ is smooth in all of $\mathbb{R}^{3}$, this gives rise to a well defined global flow.

Let $N$ be the solid torus obtained by rotating around the $z$-axis a disk of radius $r=\frac{1}{\sqrt{2}}$ centered at $(1,0,0)$ and contained in the $x z$ plane. To see whether $N$ contains some invariant structure we draw its exit set $L$ using a computer progran 4 and the criterion given by Proposition 4 If $\partial N$ is represented as a square with opposite sides identified, then $L$ is the black set depicted in Figure 4 and turns out to be closed.

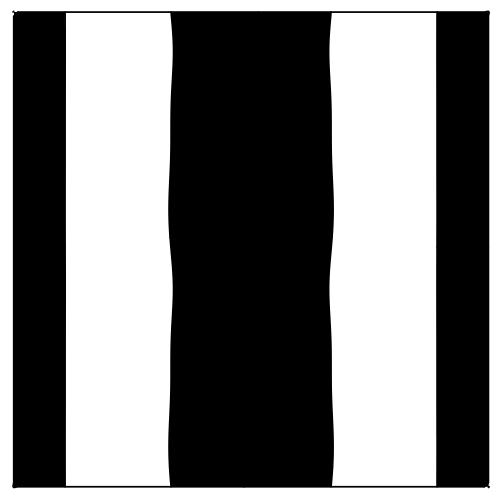

Figure 4. Exit set (in black) $L$ for the torus $N$.

Since $L$ has two path components whereas $N$ has just one, and the number of path components is a homotopy invariant, it follows that $i: L \rightarrow N$ cannot be a homotopy equivalence. Hence by Wazewski's criterion $\operatorname{inv}(N) \neq \emptyset$.

To finish this section let us introduce two notions. First of all, it will be convenient to deal exclusively with sets $N$ which are neighbourhoods of $\operatorname{inv}(N)$. These deserve a special name.

Definition 9 A compact invariant set $K$ is called isolated if it has a compact neighbourhood $N$ such that $K=\operatorname{inv}(N)$. Such a neighbourhood $N$ is called an isolating neighbourhood for $K$.

\footnotetext{
${ }^{4}$ Figure 4 was done with Mathematica 5.2.
} 
Another definition is in order, concerning the hypothesis in Theorem 5 that $L$ be closed. Although it may seem just an auxiliary condition to be able to carry out the proof of the theorem, it is indeed a crucial requirement since it is easy to find examples where $L$ is not closed and Ważewski's criterion fails. Thus we single out those well behaved sets $N$.

Definition 10 An isolating neighbourhood is called proper if its inmediate exit set is closed.

Intuitively speaking, a proper isolating neighbourhood $N$ is one which does not have interior tangencies such as that shown in case $(d)$ of Figure 3 (indeed, points to the left of $p$ would belong to the exit set, whereas $p$ itself would not, so $L$ could not be closed). We shall comment more on this notion in the following section.

\subsection{Conley's index of an isolated invariant set}

Our approach to Conley's index will take Ważewski's method as departure point. In the last section we saw how the latter could be used to detecting invariant structure inside a compact region $N$ of the phase space having a closed exit set $L$; under the assumption that $\operatorname{inv}(N)=\emptyset$ a homotopy $H$ was produced which provided a deformation retraction of $N / L$ onto $L / L$. Now we would like to examine this same situation but removing the hypothesis that $\operatorname{inv}(N)$ be empty.

The main reference for this section is Conley's monography [14], and the condensed exposition of [59]. However since we have thought it best to develop the shape theoretical version of Conley's index, because it is more straightforward and intuitively appealing, the papers [56] and [66] should also be taken into account as they contain the essentials of our approach.

To begin with, a little detour to introduce shape theory is in order.

A bRIEF REVIEW OF SHAPE theORY. We have already seen how homotopy theory enters the scene of dynamical systems in a quite straightforward way, since flows provide a natural means of constructing homotopy equivalences. However, and in a spirit similar to that which motivated our discussion of limit sets, we would like to be able to "pass to the limit", in some sense.

Example 7 Suppose that $N$ is a positively invariant compact set. The flow induces retractions $r_{t}: N \rightarrow$ $N \cdot t$ given by $r_{t}(p):=p \cdot t$, and homotopies $\operatorname{id}_{N} \simeq_{\left.H\right|_{[0, t]}} r_{t}$ for every $t \geq 0$. In particular the assertion

$$
(H E)_{t}: \text { the inclusion } j_{t}: N \cdot t \rightarrow N \text { is a homotopy equivalence }
$$

is true for every $t \geq 0$. Now the question is whether the same still holds on letting $t \rightarrow+\infty$, that is whether

$$
(H E)_{\infty} \text { : the inclusion } j: \omega(N)=N \cdot(+\infty) \rightarrow N \text { is a homotopy equivalence }
$$

is true.

Let $\mathbf{C p t}$ denote the category of compact metrizable spaces and continuous mappings between them. If $\left(P_{k}\right)_{k \in \mathbb{N}}$ is a decreasing (that is, $P_{k+1} \subseteq P_{k}$ for every $k \in \mathbb{N}$ ) sequence of compact spaces, let us call (in close analogy with the limit of a decreasing sequence of real numbers, which is their greatest lower bound) $P:=\bigcap_{k \in \mathbb{N}} P_{k}$ the limit of $\left(P_{k}\right)_{k \in \mathbb{N}}$.

Definition 11 Let $F: \mathbf{C p t} \rightarrow \mathcal{C}$ be a functor. We say that $F$ is continuous if, whenever $\left(P_{k}\right)_{k \in \mathbb{N}}$ is a decreasing sequence of compact spaces with the property that

$$
(E)_{k}: \text { the inclusion } j_{k}: P_{k} \rightarrow P_{1} \text { transforms into an equivalence under } F
$$

for every $k \in \mathbb{N}$, then the same holds for the limit inclusion:

$$
(E): \text { the inclusion } j: P \rightarrow P_{1} \text { transforms into an equivalence under } F \text {. }
$$


Example 8 The continuity theorem for Čech cohomology theory (see [70] or [71]) shows that the latter is a continuous functor $\check{H}: \mathbf{C p t} \rightarrow \mathbf{A b}$ with values in the category of graded abelian groups.

If we denote by $\mathbf{H C p t}$ the category of compact spaces and homotopy classes of continuous mappings between them, and let $\mathbf{H}: \mathbf{C p t} \rightarrow \mathbf{H C p t}$ be the functor taking each space to itself and each continuous mapping to its homotopy class, it turns out that $\mathbf{H}$ is not continuous. This issue can be overcome replacing homotopy theory by shape theory (introduced by K. Borsuk in his paper [11]), which can be thought of as some kind of continuous (or Čech type) homotopy theory.

The objects of the shape category of compact spaces SCpt are the same as those in HCpt and Cpt, just compact metrizable spaces. The morphisms are, however, a bit more complicated. Every continuous mapping $f: X \rightarrow Y$ induces a shape morphism $\mathbf{S h}(f): X \rightarrow Y$ which depends only on the homotopy class of $f$, but in general there may exist shape morphisms $u: X \rightarrow Y$ that do not come from any continuous mapping. Thus SCpt contains representatives of all the morphisms in $\mathbf{H C p t}$ plus some extra ones which account for its flexibility when it comes to comparing its objects.

Example 9 To provide the interested reader with some intuitive idea of where the extra morphisms in the shape category come from, let us describe the notion of an approximative map between two metric compacta $X$ and $Y$. Begin by assuming that $Y$ is embedded in some normed space $Q$ (this can always be done by invoking a theorem of Kuratowski and Wojdyslawski, see the appendices to [41]). An approximative map from $X$ to $Y$, denoted $f=\left\{f_{n}: X \rightarrow Y\right\}$, is a sequence of continuous mappings

$$
f_{n}: X \rightarrow Q
$$

with the property that for every neighbourhood $V$ of $Y$ in $Q$ there exists some $n_{0}$ such that

$$
f_{n} \simeq f_{m} \text { in } V \text { for } n, m \geq n_{0} .
$$

This definition (due to Borsuk) is quite appealing from the intuitive point of view, for it shows a strong resemblance with some kind of "limit" in the sense of homotopy. The reader can try his hand, in turn, at providing a suitable definition of "homotopy" between approximative maps $f, g: X \rightarrow Y$.

The reason for introducing approximative mappings is that they provide a convenient way to describe shape morphisms. Namely, the shape morphisms between $X$ and $Y$ are in correspondence with the homotopy classes of approximative maps between $X$ and $Y$ (one can check that the only arbitrary element in this construction, the embedding of $Y$ in $Q$, is immaterial.)

We shall say that two spaces $X$ and $Y$ have the same shape, and represent it by $\operatorname{Sh}(X)=\operatorname{Sh}(Y)$, if there exist two shape morphisms $u: X \rightarrow Y$ and $v: Y \rightarrow X$ which are inverses to each other, that is $v \circ u=\mathbf{S h}\left(\operatorname{id}_{X}\right)$ and $u \circ v=\mathbf{S h}\left(\operatorname{id}_{Y}\right)$. Both $u$ and $v$ are called shape equivalences.

The following theorem collects some properties of shape theory relevant to us.

Theorem 6 Let $\mathbf{S h}: \mathbf{C p t} \rightarrow \mathbf{S C p t}$ denote the functor which takes every compact space to itself and every continuous mapping to the shape morphism it induces.

1. If $u: X \rightarrow Y$ is a shape morphism between two spaces $X$ and $Y$ having the homotopy type of finite simplicial complexes (in particular if $X$ and $Y$ are compact manifolds), there exists a continuous mapping $f: X \rightarrow Y$ such that $\mathbf{S h}(f)=u$.

2. The shape functor $\mathbf{S h}$ is continuous.

3. The Čech cohomology functor factors through $\mathbf{S h}$. In particular, two spaces with the same shape have isomorphic Čech cohomology groups. 
The first assertion in Theorem 6 means that homotopy and shape theory are equivalent when the underlying spaces have the homotopy type of finite simplicial complexes. Thus shape theory, while yielding a coarser classification of spaces than homotopy theory, is still as good as it in distinguishing well behaved spaces but also enjoys the important continuity property.

The second and third parts of Theorem 6 provide a proof of Example 8

Example 10 For the setting described in Example 7 the inclusion $j: \omega(N) \rightarrow N$ is a shape equivalence. In particular $j$ induces isomorphisms $j^{*}: \check{H}^{*}(N) \rightarrow \check{H}^{*}(\omega(N))$ in Čech cohomology.

PROOF. Consider the sequence of spaces $(N \cdot k)_{k \in \mathbb{N}}$. Every $N \cdot k$ is compact because it is homeomorphic to $N$. The positive invariance of $N$ implies that $N \cdot k=(N \cdot[0,+\infty)) \cdot k=N \cdot[k,+\infty)$ and consequently

$$
k \geq l \Rightarrow N \cdot k=N \cdot[k,+\infty) \subseteq N \cdot[l,+\infty)=N \cdot l,
$$

which shows that $(N \cdot k)_{k \in \mathbb{N}}$ is a decreasing sequence. Furthermore, its limit is

$$
\bigcap_{k \in \mathbb{N}} N \cdot k=\bigcap_{k \in \mathbb{N}} \overline{N \cdot[k,+\infty)}=\omega(N) .
$$

We already stated in Example 7 that every inclusion $j_{k}: N \cdot k \rightarrow N$ is a homotopy equivalence, hence a shape equivalence too. The continuity property of the shape functorimplies then that the inclusion $j: \omega(N) \rightarrow N$ is also a shape equivalence.

Alternatively, one can find a shape inverse for the inclusion $j: \omega(N) \rightarrow N$. In the present case this turns out to be an easy task, for it is not difficult to check that the approximative map $r=\left\{r_{n}: n \in \mathbb{N}\right\}$ (recall from Example 7 that $r_{n}$ denotes the flow induced retraction at time $n$ ) provides such an inverse.

It is a well known fact that the classical tools of algebraic topology, and in particular those allowing the passage from algebraic to homotopy theoretic information (Whitehead's theorem to the effect that a weak homotopy equivalence is a homotopy equivalence may be considered a cornerstone in this sense) work best on the class of polyhedra (simplicial complexes) or, more generally, on the class of spaces having the homotopy type of polyhedra. Analogously, in shape theory the class of spaces having the shape of polyhedra (and specifically, of finite polyhedra) plays a distinguished role. It may be convenient to mention here that finite polyhedra have finitely generated Čech cohomology groups, and only finitely many of them are nontrivial, so they also have a well defined Euler characteristic. This will be of relevance later (see, for example, Theorem 12 Theorem 15 or Corollary [5].

Further information about shape theory, from many different points of view, can be found in [12], [21] and [41]. We have chosen an approach which is somewhat intermediate between Borsuk's original definition, more geometrical in nature, and that of ANR systems, introduced by S. Mardešic̀ and J. Segal ([39]). Their equivalence is shown in [40].

Let us come back to our original question, that of exploring to what extent the technique developed for Ważewski's method is informative in case $\operatorname{inv}(N) \neq \emptyset$. Observe that, if we agree to let $\tau(p)=+\infty$ for points $p \in N$ such that $p \cdot[0,+\infty) \subseteq N$, the expression defining the homotopy $H$ of the proof of Theorem 5 still makes sense. However, since $\operatorname{inv}(N)$ might not be empty, $\tau$ does not need to have an upper bound $T$ and it is reasonable to consider

$$
\begin{aligned}
H: N \times[0,+\infty) & \longrightarrow N \\
(p, t) & \longmapsto p \cdot \min \{t, \tau(p)\}
\end{aligned}
$$

It is not difficult to check that $H$ is still continuous, because $\tau$ is indeed continuous with the new, extended definition. As in the proof of Theorem 5 for every $t \geq 0$ the mapping $\left.H\right|_{[0, t]}$ provides a homotopy between $\operatorname{id}_{N}$ and $r_{t}(p):=H(p, t)$. Consequently, denoting by $\operatorname{im}\left(r_{t}\right)$ the image of $r_{t}$ (which is a compact subset of $N$, decreasing with increasing $t$ ), the inclusions

$$
j_{t}: \operatorname{im}\left(r_{t}\right) \rightarrow N
$$


are homotopy equivalences with homotopy inverse $r_{t}$. Now the continuity property of the shape functor guarantees that the inclusion

$$
j: \bigcap_{t \geq 0} \operatorname{im}\left(r_{t}\right) \rightarrow N
$$

is a shape equivalence. A moment of thought will make it clear that $p \in \bigcap_{t \geq 0} \operatorname{im}\left(r_{t}\right)$ if, and only if, either $p \in L$ or $p \cdot s \in N$ for every $s \leq 0$. Thus if we introduce the notation

$$
N^{-}:=\{p \in N: p \cdot(-\infty, 0] \subseteq N\}
$$

the intersection $\bigcap_{t>0} \operatorname{im}\left(r_{t}\right)=N^{-} \cup L$. The following Proposition (see [66]) states, finally, what happens after quotienting by $L$.

Proposition 5 Let $N$ be a proper isolating neighbourhood. Then the inclusion

$$
j:\left(\frac{N^{-}}{n^{-}}, \frac{n^{-}}{n^{-}}\right) \longrightarrow\left(\frac{N}{L}, \frac{L}{L}\right)
$$

is a shape equivalence, where $n^{-}:=N^{-} \bigcap \partial N$.

ProOF. We keep the notation of the argument above. Since $H$ keeps points in $L$ fixed (because $\tau(p)=0$ when $p \in L$ ), the pair inclusion

$$
\left(N^{-} \bigcup L, L\right) \longrightarrow(N, L)
$$

is a shape equivalence, and on passing to the quotient by $L$ we get that

$$
\left(\frac{N^{-} \cup L}{L}, \frac{L}{L}\right) \longrightarrow\left(\frac{N}{L}, \frac{L}{L}\right)
$$

is a shape equivalence too.

Now observe that collapsing $L$ to a point in $N^{-} \bigcup L$ is the same as collapsing $N^{-} \bigcap L$ to a point in $N^{-}$. Furthermore, we assert that $N^{-} \bigcap L=n^{-}$. Indeed, for any $p \in N^{-} \cap \partial N$ we have $p \cdot(-\infty, 0] \subseteq N$, and if $p$ did not belong to $L$ then for some $\varepsilon>0$ the inclusion $p \cdot[0, \varepsilon] \subseteq N$ would hold. Thus $p \cdot(-\infty, \varepsilon)$ would be a trajectory segment contained in $N$ but meeting $\partial N$ at $p$, that is, $p$ would be an interior tangency point for $N$, which contradicts the hypothesis that $N$ is a proper isolating neighbourhood. Hence $N^{-} \bigcap \partial N \subseteq L$ and consequently $N^{-} \bigcap L=N^{-} \bigcap \partial N=n^{-}$, so

$$
\left(\frac{N^{-} \bigcup L}{L}, \frac{L}{L}\right) \cong\left(\frac{N^{-}}{N^{-} \bigcap L}, \frac{L}{L}\right)=\left(\frac{N^{-}}{n^{-}}, \frac{n^{-}}{n^{-}}\right)
$$

and the proof is finished.

A fundamental consequence of Proposition 5 is that the $\operatorname{Sh}(N / L, L / L)$ does not depend on $N$, but only on $K:=\operatorname{inv}(N)$.

Corollary 3 Let $N_{1}$ and $N_{2}$ be proper isolating neighbourhoods for $K$. Then

$$
\operatorname{Sh}\left(N_{1}^{-}, n_{1}^{-}\right)=\operatorname{Sh}\left(N_{2}^{-}, n_{2}^{-}\right)
$$

and consequently

$$
\operatorname{Sh}\left(\frac{N_{1}^{-}}{n_{1}^{-}}, \frac{n_{1}^{-}}{n_{1}^{-}}\right)=\operatorname{Sh}\left(\frac{N_{2}^{-}}{n_{2}^{-}}, \frac{n_{2}^{-}}{n_{2}^{-}}\right)
$$


Proof. Observe that both $N_{1}^{-}$and $N_{2}^{-}$are negatively invariant sets such that $\alpha\left(N_{1}^{-}\right)=\alpha\left(N_{2}^{-}\right)=K$, so by Example 10 (or rather a dual version of it) the inclusions $j_{1}: K \rightarrow N_{1}^{-}$and $j_{2}: K \rightarrow N_{2}^{-}$are shape equivalences. Thus $\operatorname{Sh}\left(N_{1}^{-}\right)=\operatorname{Sh}\left(N_{2}^{-}\right)$. We leave it to the reader to modify the argument in Example [10]to obtain the shape equality of pairs stated in the proposition. The assertion on the quotients follows from [38].

The following definition is now justified:

Definition 12 Let $K$ be an isolated invariant set. The shape index of $K$ is the shape

$$
s(K):=\operatorname{Sh}\left(\frac{N}{L}, \frac{L}{L}\right),
$$

where $N$ is any proper isolating neighbourhood for $K$.

Conley defined the homotopy index $h(K)$ of an isolated invariant set $K$ as the homotopy type of the pair $(N / L, L / L)$, where $N$ is any proper isolating neighbourhood for $K$. Thus our definition is just a shape theoretical version of Conley's. However, the proof that $h(K)$ does not depend on the particular $N$ is a bit involved, and this is one of the reasons why we chose this approach to present Conley's index.

Neither the homotopy nor the shape index are very well suited for computational purposes, where either the cohomological Conley index or the homological Conley index are preferred instead. For example, the former is defined as the Čech cohomology module of $(N / L, L / L)$, that is $C \breve{H}(K):=\check{H}(N / L, L / L)$, and the latter is defined similarly. The cohomological index has the advantage that, due to the continuity of Čech cohomology theory, $C \check{H}(K)=\check{H}(N, L)$.

There is a theorem of Mrozek [51] to the effect that, if the phase space $M$ is a manifold (or more generally if it has the homotopy type of a finite polyhedron, see [10], [36] or [41]), the pair $(N / L, L / L)$ has finitely generated Čech cohomology groups, and only finitely many of them are nontrivial. Hence one can also define the Conley-Euler index of $K$, denoted by $\chi(K)$, as $\chi s(K):=\chi(N, L)$.

Now let us carry out some calculations.

Example 11 Observe that Ważewski's criterion inmediately implies that $s(\emptyset)=\operatorname{Sh}(*, *)$ because if $N$ is an proper isolating neighbourhood for the empty set, by Corollary 2 the pair $(N / L, L / L)$ is homotopy equivalent (hence shape equivalent, too) to $(*, *)$.

Example 12 Coming back to Example 6 we can easily compute the Conley index of $K:=\operatorname{inv}(N)$. Indeed, $N$ is a solid torus and $L$ is the disjoint union of two annuli contained in $\partial N$, so $N / L$ is homotopy equivalent to the wedge sum $\mathbb{S}^{1} \vee \mathbb{S}^{1} \vee \mathbb{S}^{2} \vee \mathbb{S}^{2}$.

To be able to compute the shape index of a fixed point for a differential equation we need to recall some relevant notions.

A BRIEF REVIEW OF HYPERBOLIC FIXED POINTS. Let

$$
(E): \dot{p}(t)=f(p(t))
$$

be a differential equation in some manifold $M$, which we shall assume for simplicity to be $\mathbb{R}^{n}$. Suppose that $p_{0}$ is a fixed point for $(E)$, that is $f\left(p_{0}\right)=0$. Then $f(p)=D_{p_{0}} f \cdot\left(p-p_{0}\right)+o\left(\left\|p-p_{0}\right\|\right)$ and it is reasonable to ask to what extent the linearized equation

$$
(L E): \dot{p}(t)=D_{p_{0}} f \cdot\left(p(t)-p_{0}\right)
$$

provides a good aproximation to $(E)$ near $p_{0}$.

It turns out that the key notion here is that of hyperbolicity. 
Definition 13 The fixed point $p_{0}$ for equation $(E)$ is hyperbolic if the matrix $D_{p_{0}} f$ has no eigenvalues with zero real part.

It is not easy to justify this definition in a few words, and we shall not attempt to do it. However, the significance of this notion can be ascertained by the following very important result.

Theorem 7 (Hartman-Grobman) Let $p_{0}$ be a hyperbolic fixed point for $(E)$. Then, in a sufficiently small neighbourhood of $p_{0}$, the flow generated by $(E)$ is equivalent to that generated by the linearized equation $(L E)$.

Thus in the proximities of a hyperbolic fixed point the phase portrait for $(E)$ looks like that of $(L E)$ which, being a linear system, can be solved explicitly with elementary means and is well understood.

Proposition 6 Let $p_{0}$ be a hyperbolic fixed point for $(E)$. Possibly after a change of coordinates, there exist two closed disks $D^{s} \subseteq p_{0}+\mathbb{R}^{\ell} \times\{0\}$ and $D^{u} \subseteq p_{0}+\{0\} \times \mathbb{R}^{n-\ell}$, where $\ell$ is the number of eigenvalues of $D_{p_{0}} f$ with negative real part, such that

1. $N:=D^{s} \times D^{u}$ is a compact neighbourhood of $p_{0}$.

2. $p \in N$ belongs to $D^{s} \Leftrightarrow p \cdot[0,+\infty) \subseteq N$,

3. $p \in N$ belongs to $D^{u} \Leftrightarrow p \cdot(-\infty, 0] \subseteq N$.

The disks $D^{s}$ and $D^{u}$ are called the local stable and local unstable manifolds of $p_{0}$. Observe that if a point $p \in N$ is not in $D^{s} \cup D^{u}$, then its trajectory exits $N$ both in the future and in the past.

Proof. Use the Hartman-Grobman theorem to obtain a neighbourhood $U$ of $p_{0}$ where $(E)$ is equivalent to $(L E)$. Now show that there exist disks $E^{s}$ and $E^{u}$ for $(L E)$ which satisfy all three conditions in the statement of the proposition together with $E^{s} \times E^{u} \subseteq U$.

Figure 5 depicts three phase portraits, corresponding to three linear systems for which the origin $p_{0}=0$ is a fixed point. In case $(a) D^{s}=N$ can be taken to be a ball centered at the origin, and $D^{u}=\left\{p_{0}\right\}$; whereas in $(b)$ we have marked $D^{s}$ and $D^{u}$, both of dimension one. The system shown in $(c)$ does not have the origin as a hyperbolic point.

Proposition 6 can be strenghtened to prove that $D^{s}$ and $D^{u}$ are differentiable manifolds (a result known as the stable manifold theorem) of the same class as $f$. However this is quite involved and we will not need it (see [31], [69] or [74]).

Example 13 Let $p_{0}$ be a hyperbolic equilibrium for the differential equation $(E): \dot{p}(t)=f(p(t))$. The neighbourhood $N$ of $p_{0}$ whose existence was proved in Proposition 6 is in fact a proper isolating neighbourhood (this is easy to see from the way it was constructed). Now parts 2 and 3 of the same proposition imply that $N^{-}=D^{u}$ is a $k$-dimensional disk, where $k$ is the number of positive eigenvalues of $D_{p_{0}} f$, and $n^{-}=\partial D^{u}=\mathbb{S}^{k-1}$ is its boundary, a $k$-dimensional sphere. Hence $N^{-} / n^{-}$has the shape (and the homotopy type) of a $k$-dimensional sphere and consequently $s\left(\left\{p_{0}\right\}\right)=\operatorname{Sh}\left(\mathbb{S}^{k}, *\right)$.

This last example may shed some light about the interpretation of $N^{-}$and its positive counterpart $N^{+}:=\{p \in N: p \cdot[0,+\infty) \subseteq N\}$. They can be thought of as the analogues of the local unstable and stable manifolds $D^{u}$ and $D^{s}$ respectively, when $N$ is a proper isolating neighbourhood for a compact invariant set $K$. Poincaré, Smale and Conley's indices all measure, in some sense, the size of these local unstable manifolds.

The shape index is defined only for invariant sets having a proper isolating neighbourhood, and it is therefore natural to ask what invariant sets satisfy this requirement. The following definition and theorem provide the answer (proofs can be found in [15] and [17]).

Definition 14 An isolating block for a compact invariant set $K$ is an isolating neighbourhood such that every $p \in \partial N$ is of one the types $(a),(b)$ or $(c)$ shown in Figure 3 


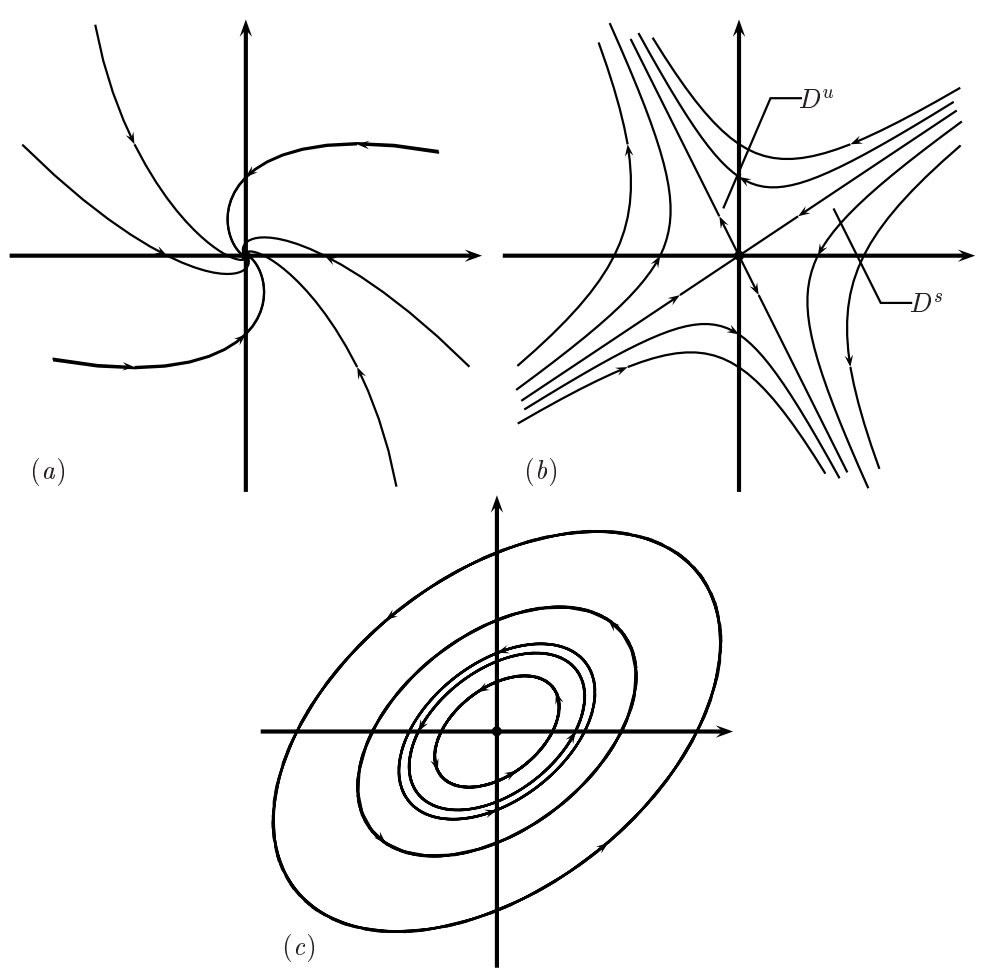

Figure 5. Phase portrait of three linear systems.

Every isolating block is a proper isolating neighbourhood, but the converse is not true. However, the existence of a single isolating neighbourhood for $K$ guarantees that plenty of isolating blocks exist:

Theorem 8 Let $K$ be an isolated invariant set. Then K has a neighbourhood basis comprised of isolating blocks. Furthermore, if the flow is differentiable, those isolating blocks can be chosen to be differentiable manifolds which contain $L$ as a submanifold of their boundaries.

In practice, checking whether a compact set $N$ is an isolating neighbourhood would require to ascertain if the trajectory of every $p \in \partial N$ exits $N$ either in the future or in the past, which can be extremely complicated. On the other hand, it is an easy matter (at least in the case of flows coming from differential equations) to see if there are no interior tangencies to $\partial N$, using criteria similar to those developed in Proposition 4 and in that case $N$ is not only an isolating neighbourhood, but also an isolating block (this is what we did in Example 6. Hence the importance of Theorem 8 is more of a theoretical nature, and in view of this we shall not examine its interesting proof.

CONLEY'S INDEX IN SURFACES. We stated above that Conley's work could be inscribed in a line of thought dating back to Poincare and his works about flows on surfaces, and the following lines are devoted to a short exploration of this relation. Thus for this digression we shall concentrate on continuous flows on surfaces, where a surface means a 2-manifold (either compact or not, with boundary or not) and a closed surface means a compact 2-manifold without boundary. The following result, known as Gutierrez's smoothing theorem (see [30]), will be very useful:

Theorem 9 Every continuous flow on a closed surface $M$ is conjugate to a differentiable flow. 
As a corollary we have

Corollary 4 Let $K$ be an isolated invariant set for a continuous flow $\varphi$ in a surface $M$. Then $K$ has a neighbourhood basis of isolating blocks $N$ which are compact 2-manifolds with boundary $\partial N$ and such that their exit sets $L$ are compact 1 -submanifolds of $\partial N$.

PROOF. If the surface $M$ were closed, then the result would follow inmediately from Theorem 8 and Theorem 9 In the general case, it is clear that is suffices to embed $K$, together with a neighbourhood of it and the flow it carries, into some closed surface and then apply the smoothing theorem there. To this end, let $P$ be a compact 2-manifold with boundary $\partial P$ such that $i n v(P)=K$ (just let $P$ be a compact manifold containing $K$ in its interior and contained in any isolating neighbourhood of $K$ ). By an easy modification of a theorem of Beck [5] a new flow $\psi$ can be obtained in $M$ which leaves every point in $\partial P$ fixed and is locally equivalent to $\varphi$ on $\operatorname{int}(P)$. It only remains to collapse every component of $\partial P$ to a single point to obtain a closed surface $\widehat{P}$ and a flow $\widehat{\psi}$ which contain an embedded copy of $K$ and its open neighbourhood $\operatorname{int}(P)$, together with the trajectory segments it carries.

From now on we shall assume that all isolating blocks are chosen to be of the form described in Corollary 4 Thus if $K$ is an isolated invariant set and $N$ is an isolating block for $K$, its exit set $L$ is the disjoint union of a finite number of intervals $J_{1}, \ldots, J_{m}$ and circumferences $C_{1}, \ldots, C_{n}$. With this notation,

$$
\chi s(K)=\chi(N, L)=\chi(N)-\sum_{k=1}^{m} \chi\left(J_{k}\right)-\sum_{\ell=1}^{n} \chi\left(C_{\ell}\right)=\chi(N)-m
$$

and consequently we can discard the components $C_{1}, \ldots, C_{n}$, which do not contribute to $\chi s(K)$, to concentrate on $J_{1}, \ldots, J_{m}$. Observe that the endpoints of each of these must be exterior tangency points, so every consecutive pair of those determines precisely one of the components $J_{k}$. Hence

$$
\chi s(N)=\chi(N)-\frac{1}{2} E,
$$

where $E$ is the number of exterior tangencies to $N$.

Poincaré assigned to every isolated fixed point a numerical index $\iota(p)$ (now called its Poincaré-Hopf index). A fixed point is isolated if it has a neighbourhood which does not contain any more fixed points. An isolated fixed point need not be an isolated invariant set, think for example of the origin in Figure $5(c)$.

Definition 15 Let $p$ be an isolated fixed point and let $D$ be a closed disk which contains $p$ (no other fixed points) and satisfies the property that every point in $\partial D$ is of one of the four types described in Figure 3 The Poincaré index of $p$ is the integer

$$
\iota(p):=1+\frac{1}{2}(I-E),
$$

where I and $E$ denote the number of interior and exterior tangencies to $D$ (cases $(d)$ and (c) of Figure 3 respectively).

Poincare shows that the definition is independent of the disk $D$ chosen and assumes that it is always possible to find such a $D$ (it indeed is). Then he continues on to state and prove the celebrated Poincaré-Hopf which we already saw above (Theorem 3]. With these tools we can compare the Poincaré and Conley-Euler indices as follows.

Theorem 10 Let $K$ be an isolated invariant set for a flow in a 2-manifold $M$. Suppose that $K$ contains finitely many fixed points $p_{1}, \ldots, p_{m}$. Then

$$
\sum_{k=1}^{m} \iota\left(p_{k}\right)=\chi s(K)
$$


Proof. By Corollary $4 K$ has an isolating block $N^{\prime}$ which is a compact 2-manifold with boundary. We shall denote the boundary components (a finite number of circumferences) by $C_{1}^{\prime}, \ldots, C_{n}^{\prime}$. Let $N$ be the result of enlarging $N^{\prime}$ by attaching a small closed external collar to it. Here "small" means sufficiently small so that $N$ is still an isolating neighbourhood of $K$ (in fact, if the choice of the collar is made in a sensible way, $N$ will also be an isolating block for $K$, but we do not need this). We denote the boundary components of $N$ by $C_{1}, \ldots, C_{n}$, each $C_{\ell}$ corresponding to $C_{\ell}^{\prime}$.

Using the same trick as in Corollary 4 the flow in $M$ can be substituted by another one $\psi$ which is locally equivalent to the former in $\operatorname{int}(N)$ and $M-N$ but leaves all points in $\partial N$ fixed. Thus we can collapse, in $N$, every component $C_{k}$ of $\partial N$ to a single point $c_{k}$ to obtain a closed 2-manifold $\widehat{N}$ which carries a well defined flow $\widehat{\psi}$. The latter is locally equivalent to $\varphi$ in $\widehat{N}-\left\{c_{1}, \ldots, c_{n}\right\} \cong \operatorname{int}(N)$ and leaves $c_{1}, \ldots, c_{n}$ fixed.

Applying the Poincaré-Hopf theorem to $\widehat{\psi}$ and having in mind that its fixed points are precisely $p_{1}, \ldots$, $p_{m}$ and $c_{1}, \ldots, c_{n}$ we get the equality

$$
\sum_{k=1}^{m} \iota\left(p_{k}\right)+\sum_{\ell=1}^{n} \iota\left(c_{\ell}\right)=\chi(\widehat{N}) .
$$

To determine $\chi(\widehat{N})$ observe that the net result of collapsing each of $C_{1}, \ldots, C_{n}$ to a single point $c_{1}, \ldots, c_{n}$ is equivalent to capping them off with disks, so $\chi(\widehat{N})=\chi(N)+n$ and consequently $\chi(\widehat{N})=\chi\left(N^{\prime}\right)+n$, because the inclusion $N^{\prime} \rightarrow N$ is a homotopy equivalence. Hence

$$
\sum_{k=1}^{m} \iota\left(p_{k}\right)+\sum_{\ell=1}^{n} \iota\left(c_{\ell}\right)=\chi\left(N^{\prime}\right)+n .
$$

It only remains to compute the indices $\iota\left(c_{\ell}\right)$. If $A_{\ell}$ denotes the annulus comprised between $C_{\ell}$ and the corresponding boundary component $C_{\ell}^{\prime}$ of $N^{\prime}$, its projection onto $\widehat{N}$ gives a disk $D_{\ell}$ which isolates the fixed point $c_{\ell}$ from the remaining ones and, moreover, the interior (resp. exterior) tangencies to $\partial D_{\ell}$ are precisely the exterior (resp. interior) tangencies to $C_{\ell}^{\prime}$. Since $N^{\prime}$ is an isolating block, it does not have interior tangencies, so denoting $E_{\ell}^{\prime}$ the number of exterior tangencies to $N^{\prime}$ on $C_{\ell}^{\prime}$ it follows that $\iota\left(q_{\ell}\right)=1+\frac{1}{2} E_{\ell}^{\prime}$. Substituting this in the formula above we get

$$
\sum_{k=1}^{m} \iota\left(p_{k}\right)+n+\frac{1}{2} \sum_{\ell=1}^{n} E_{\ell}^{\prime}=\chi\left(N^{\prime}\right)+n,
$$

and observing that $\sum_{\ell=1}^{n} E_{\ell}^{\prime}$ is the total number $E^{\prime}$ of exterior tangencies to $N^{\prime}$ it follows that

$$
\sum_{k=1}^{m} \iota\left(p_{k}\right)=\chi\left(N^{\prime}\right)-\frac{1}{2} E^{\prime}
$$

which finishes the proof since we already argued at the beginning of this discussion that the right hand side of the formula above equals $\chi s(K)$.

Example 14 From Theorem 10 follows inmediately that if $K$ is an isolated invariant set for a flow in a 2 -manifold and $\chi s(K) \neq 0$, then $K$ contains at least one fixed point.

Theorem 10 is also true in higher dimensions provided the flow is differentiable. See [43] for further references. 


\subsection{Morse decompositions and the Morse equations}

Once the Conley index (in its shape theoretical version) has been developed, we are in conditions to present the Morse equations, which relate the local dynamical information captured by the shape indices of some family $\mathcal{K}$ of disjoint compact invariant sets and the global topology of $M$. For the rest of this discussion, the phase $M$ will be assumed to be compact. Under this assumption, and if we want the members of $\mathcal{K}$ to be isolated, we are led to consider only families $\mathcal{K}$ which are finite (otherwise the sets in $\mathcal{K}$ would accumulate).

Definition 16 A Morse decomposition of $M$ is a family $\mathcal{M}=\left\{M_{1}, \ldots, M_{m}\right\}$ of disjoint compact invariant sets which admits a Lyapunov function $G$. The labeling of the Morse sets $M_{j}$ will always be chosen in such a way that the values $c_{j}$ that $G$ assumes on them are increasing, $c_{1}<\cdots<c_{m}$.

The compactness of $M$ and Proposition 3 above guarantee that, if $\mathcal{M}$ is a Morse decomposition of $M$, every $p \notin \bigcup_{j=1}^{m} M_{j}$ has $\emptyset \neq \alpha(p) \subseteq M_{s}$ and $\emptyset \neq \omega(p) \subseteq M_{r}$ for some indices $1 \leq r<s \leq m$. In fact it can be proved (see for example [14], [59]) that any family of compact disjoint invariant sets $\mathcal{M}$ which satisfies the properties we have just mentioned is a Morse decomposition of $M$ (that is, there is a Lyapunov function for $\mathcal{M}$ ). Thus one can define what a Morse decomposition is without mentioning Lyapunov functions at all, and this is in fact the standard approach.

Example 15 If, in Example 4 each critical point of $f$ is assumed to be isolated, then there exist only finitely many of them $p_{1}, \ldots, p_{m}$ and $\mathcal{M}=\left\{p_{1}, \ldots, p_{m}\right\}$ is a Morse decomposition of $M$ (maybe after a suitable relabeling of the critical points) because $f$ is a Lyapunov function for $\mathcal{M}$.

Example 16 A celebrated theorem of Peixoto [57] implies that every flow in a closed orientable surface $M$ can be approximated as closely as desired (in some sense which we will not be precise about) by another flow for which the chain recurrent set (see Theorem 4) has finitely many connected components (these being hyperbolic fixed points and periodic orbits), the collection of which constitutes a Morse decomposition of $M$. Since it is generally agreed that the interesting behaviour of a dynamical system is that which survives under small perturbations (although this is quite convincingly questioned in [28]), this would imply that flows having a Morse decomposition are enough to understand continuous dynamical systems in closed orientable surfaces.

The following result shows that Morse sets are indeed isolated, so that they can be assigned their shape index.

Proposition 7 Every Morse set in a Morse decomposition $\mathcal{M}=\left\{M_{1}, \ldots, M_{m}\right\}$ is an isolated invariant set.

PROOF. With the notations of Definition 16 pick for $1 \leq k \leq m$ a number $\varepsilon_{k}>0$ sufficiently small such that $\left[c_{k}-\varepsilon, c_{k}+\varepsilon\right]$ does not contain any other critical value of $G$ and let $N_{k}:=G^{-1}\left[c_{k}-\varepsilon, c_{k}+\varepsilon\right]$. Since $M_{k}$ is compact and $M$ is locally compact, $N_{k}$ is compact if $\varepsilon_{k}>0$ is chosen small enough. Now suppose $p \notin M_{k}$ satisfies $p \cdot \mathbb{R} \subseteq N_{k}$ and observe that then its $\alpha$ - and $\omega$-limits are nonempty subsets of $N_{k}$ (see Example 5). Since $p \notin \bigcup_{j=1}^{m} M_{j}$ (otherwise $p \in M_{k}$, but we assumed the contrary) it follows by Proposition 3 that $c_{k}=\left.G\right|_{\alpha(p)}>G(p)>\left.G\right|_{\omega(p)}=c_{k}$, which is a contradiction. Thus $N_{k}$ isolates $M_{k}$ (in fact it is straightforward to see that $L_{j}:=G^{-1}\left(c_{k}+\varepsilon\right)$ is the exit set for $N_{k}$, which is closed, so $N_{j}$ is an isolating block for $M_{k}$ ).

An analogue of the Poincaré-Hopf theorem is very easily obtained in this context.

Proposition 8 Let $\mathcal{M}=\left\{M_{1}, \ldots, M_{m}\right\}$ be a Morse decomposition such that every $M_{j}$ has a well defined Conley-Euler index (for example, if $M$ is a manifold or, more generally, has the homotopy type of a compact polyhedron). Then

$$
\sum_{j=1}^{m} \chi s\left(M_{j}\right)=\chi(M) .
$$


Proof. Let $G$ be a Lyapunov function for $\mathcal{M}$. We can assume, without loss of generality, that $G\left(M_{j}\right)=j$ for $1 \leq j \leq m$. Each $N_{j}:=G^{-1}\left[j-\frac{1}{2}, j+\frac{1}{2}\right]$ is an isolating block for $K_{j}$ with exit set $L_{j}=G^{-1}\left[j+\frac{1}{2}\right]$ (see Proposition 7). Observe that the pairwise intersections $N_{j} \bigcap N_{k}$ are empty unless $k=j+1$ (or viceversa), and in that case $N_{j} \cap N_{k}=L_{j}$, and that the intersection of any three distinct $N_{j}^{\prime} s$ is empty. Thus, since $M=\bigcup_{j=1}^{m} N_{j}$, we have

$$
\chi(M)=\chi\left(\bigcup_{j=1}^{m} N_{j}\right)=\sum_{j=1}^{m} \chi\left(N_{j}\right)-\sum_{j=1}^{m} \chi\left(L_{j}\right)=\sum_{j=1}^{m} \chi\left(N_{j}, L_{j}\right)=\sum_{j=1}^{m} \chi s\left(K_{j}\right) .
$$

This completes the proof.

Example 17 Let $\mathcal{M}=\left\{p_{1}, \ldots, p_{m}\right\}$ be a Morse decomposition of the closed surface $M$ whose Morse sets are all hyperbolic fixed points. Then $\mathcal{M}$ contains precisely $\alpha$ attractors or repellers and $\beta$ saddle points, where

$$
\alpha=\frac{m+\chi(M)}{2}, \quad \beta=\frac{m-\chi(M)}{2} .
$$

Furthermore, $m$ has the same parity of $\chi(M)$ and $m \geq \chi(M)$.

PROOF. Let $k_{j}$ be the dimension of the unstable manifold of $p_{j}$. It was shown in Example 13 that $s\left(p_{j}\right)=$ $\mathrm{Sh}\left(\mathbb{S}^{k_{j}}, *\right)$, so $\chi s\left(M_{j}\right)=(-1)^{k_{j}}$ and by Proposition 8 we must have

$$
\sum_{j=1}^{m}(-1)^{k_{j}}=\chi(M)
$$

Now observe that cases $k_{j}=0,2$ correspond to attractors and repellers respectively, while $k_{j}=1$ corresponds to saddle points. Therefore substituting in the formula above we get $-\beta+(m-\beta)=\chi(M)$, or $\beta=\frac{m-\chi(M)}{2}$ and $\alpha=m-\beta=\frac{m+\chi(M)}{2}$. The lower bound for $m$ and the assertion about its parity follow from the fact that $\beta$ is a non negative integer.

Proposition 8 shows a specific instance of a much more general formula relating the shape indices of the Morse sets to the Betti numbers of $M$. To state it we need to introduce some notation: we put

$$
p\left(s\left(M_{j}\right) ; t\right):=\sum_{k=0}^{\infty} \operatorname{rk} \check{H}^{k}\left(N_{j}, L_{j}\right) \cdot t^{k},
$$

which is a formal polynomial whose $k$-th coefficient is just the rank of the $k$-th cohomology group of the pair $s\left(M_{j}\right)$ (it may be infinite), and similarly we let

$$
p(M ; t):=\sum_{k=0}^{\infty} \operatorname{rk} \check{H}^{k}(M) \cdot t^{k} .
$$

Theorem 11 (Morse equations) If $\mathcal{M}=\left\{M_{1}, \ldots, M_{m}\right\}$ is a Morse decomposition of the compact phase space $M$, then

$$
\sum_{j=1}^{m} p\left(s\left(M_{j}\right) ; t\right)=p(M ; t)+(1+t) q(t),
$$

where $q(t)$ is a polynomial with nonnegative integer coefficients 5

For a proof of Theorem 11 see, for example [16]. We will not give one here because it is basically a moderately difficult exercise in algebraic topology but does not involve any relevant constructions with flows whatsoever, and therefore is uninteresting for us.

\footnotetext{
${ }^{5}$ It is known that the polynomial $q(t)$ measures, in some sense, the amount of connecting orbits between the Morse sets, although a completely satisfactory dynamical interpretation for it has not been yet found. This subject is too complex to give even a simple description here, but the papers $22,24,42$ and 60 contain relevant information.
} 
MORSE THEORY. Let us come back briefly, as a particular case of Theorem 11 to Morse theory. We shall keep the notations of Example 4 and Example 15 If $p_{0}$ is such a critical point for $f$ and the hessian of $f$ at $p_{0}$ is nondegenerate, the second order approximation

$$
f(p)=f\left(p_{0}\right)+\left(p-p_{0}\right)^{T} \cdot H_{p_{0}} f \cdot\left(p-p_{0}\right)+o\left(\left\|p-p_{0}\right\|^{2}\right)
$$

is good enough to be able to describe the local aspect of $f$ near $p_{0}$ (this is done via a result known as Morse's lemma) and in particular allows one to conclude that the dimension of the local unstable manifold of $p_{0}$ (see Proposition 6 is precisely the number of negative entries in the canonical diagonal form of the nondegenerate quadratic form $H_{p_{0}} f$ (which is an invariant of $H_{p_{0}} f$ by Sylvester's law of inertia).

Definition $17 f$ is a Morse function if its hessian is nondegenerate at every critical point. The Morse index $m(p)$ of such a critical point $p$ is the number of negative entries in the canonical diagonal form of $H_{p} f$.

If $f$ is a Morse function, then there exist only finitely many equilibria $\left\{p_{1}, \ldots, p_{m}\right\}$, which are hyperbolic fixed points, and they constitute a Morse decomposition of $M$ (this is Example 15). By Example 13 and the discussion above we have $p\left(s\left(p_{k}\right) ; t\right)=t^{m\left(p_{j}\right)}$ for every $1 \leq k \leq m$ and consequently

$$
\sum_{j=1}^{m} t^{m\left(p_{j}\right)}=p(M ; t)+(1+t) q(t)
$$

with $q$ a non negative polynomial. Denoting by $\beta_{k}$ the number of fixed points $p_{j}$ with Morse index $m\left(p_{j}\right)=k$ we can collect terms in the summation above to get Morse's equation

$$
\sum_{k=1}^{n} \beta_{k} t^{k}=p(M ; t)+(1+t) q(t)
$$

(recall that $n$ is the dimension of $M$ ). Morse's inequalities follow by expanding the polynomial identity and using the nonnegativeness of the $q(t)$.

Let us present a pair of examples to illustrate how the above equality can be put into use.

Example 18 Let $M$ be connected and orientable, and suppose there exist just two fixed points $p_{1}$ and $p_{2}$. Our assumptions on $M$ imply that $p(M ; t)=1+a_{1} t+\cdots+a_{n-1} t^{n-1}+t^{n}$. Now in Morse's equation

$$
t^{m\left(p_{1}\right)}+t^{m\left(p_{2}\right)}=1+a_{1} t+\cdots+a_{n-1} t^{n-1}+t^{n}+(1+t) q(t)
$$

the left hand side has exactly two nonzero terms, and the positivity of q implies that the right hand side has at least two nonzero terms, namely the lowest and highest degree ones. Hence $a_{1}=\cdots=a_{n-1}=0$ and $m\left(p_{1}\right)=0, m\left(p_{2}\right)=n$ (or viceversa). In particular $p_{1}$ is an attracting point, $p_{2}$ is a repelling point and $M$ is a homology sphere (it can be shown that $M$ is indeed a sphere, see [45]).

Example 19 Now suppose that $M$ is the 2-torus. Then Morse's equation reads

$$
\beta_{0}+\beta_{1} t+\beta_{2} t^{2}=1+2 t+t^{2}+(1+t) q(t),
$$

and letting $q(t)=q_{0}+q_{1}$ ( (observe that $q$ cannot have higher degree terms because then the right hand side of the equality above would have degree three or higher, whereas the left hand side is of degree at most two) one readily calculates that

$$
\beta_{0}=1+q_{0}, \quad \beta_{1}=2+q_{0}+q_{1}, \quad \beta_{2}=1+q_{1}
$$

with $q_{0}, q_{1} \geq 0$, or in other terms

$$
\beta_{0} \geq 1, \quad \beta_{2} \geq 1, \quad \beta_{1}=\beta_{0}+\beta_{2} \geq 2 .
$$


Expositions of Morse theory can be found in [34] and [45]. Morse theory has also been exceedingly relevant in the realm of differential topology, providing an alternative approach to the all-important tool of handle theory (see, for example, Milnor's book [47]).

A natural enough idea which may have occurred to the reader is to represent a Morse decomposition $\mathcal{M}:=\left\{M_{1}, \ldots, M_{m}\right\}$ by a graph having a vertex $v_{j}$ for each Morse set $M_{j}$ and a directed edge from $v_{s}$ to $v_{r}$ whenever there exist connecting orbits from $M_{s}$ to $M_{r}$. This idea was formalized in a somewhat refined version by Franks [23] (see also [19]) and subsequently enriched by decorating the graph with homological information concerning the indices of the Morse sets. The latter version and some applications can be found in [6] and references therein.

To finish this section let us remark that the homological relation between the indices of the Morse sets and the Betti numbers of the phase space $M$ summarized in Theorem 11 can be strenghtened. Assuming that $M$ is a manifold, the papers [7], [18], [20] include several procedures which, under appropriate circumstances, can lead to the recovery of the topological type of $M$ using the Conley indices of a Morse decomposition of $M 6$

\section{An example: the topology of attractors}

This final section presents some results about attractors which can be inscribed in the line of thought described in this survey. Since we feel that the essentials of the geometric theory of dynamical systems have probably been already grasped by the reader, we shall not present nearly any proofs. If the preceding section contained a finished theory, this one is of a more sketchy nature and does not intend to be complete by any means.

Let $K$ be a compact invariant set. A point $p \in M$ is said to be attracted by $K$ if $p \cdot t$ approaches $K$ when $t \rightarrow+\infty$ (that is, for every neighbourhood $U$ of $K$ there exists some $t_{0}$ such that $p \cdot t \in U$ for every $t \geq t_{0}$ ). This can alternatively be expressed in terms of the $\omega$-limit of $p$, since it is equivalent to requiring that $\emptyset \neq \omega(p) \subseteq K$.

Definition 18 A compact invariant set $K$ is an attractor if its basin of attraction

$$
\mathcal{A}(K):=\{p \in M: p \text { is attracted by } K\}
$$

is a neighbourhood of $K$.

In Figure $6(a)$, the point $q$ is an attractor with basin $\mathbb{R}^{2}-\{p\}$ (this famous example is due to Mendelson [44]) whereas in (b) the whole periodic orbit $\gamma$ is an attractor with basin $\mathbb{R}^{2}-\{p\}$. Figure 5 (a) depicts a flow where the origin is a global attractor, this meaning that its basin of attraction is the whole phase space.

Observe that $\mathcal{A}(K)$ is invariant, since $\omega(p \cdot t)=(p \cdot t) \cdot(+\infty)=p \cdot(t+\infty)=p \cdot(+\infty)=\omega(p)$ for every $p \in M$ and $t \in \mathbb{R}$. Moreover, it is an open set. To see this, suppose not and pick some $p \in \partial \mathcal{A}(K)$ such that $p \in \mathcal{A}(K)$, so $\emptyset \neq \omega(p) \subseteq K$. However $\partial \mathcal{A}(K)$ is invariant (being the boundary of an invariant set) and closed so $\omega(p) \subseteq \partial \mathcal{A}(K)$, which implies that $\emptyset \neq \omega(p) \subseteq K \cap \partial \mathcal{A}(K)$, contradicting the fact that $\mathcal{A}(K)$ is a neighbourhood of $K$.

Since every initial condition close enough to an attractor $K$ will eventually approach it, $K$ can be considered as representative of the long term behaviour of the flow. However it may very well happen that points in its basin of attraction, even arbitrarily close to $K$, take a very long time to get anywhere near the attractor (for instance, in Figure 6(a) there are points in $\gamma$ which start very near the attractor $q$ but have to traverse the whole unit circumference before coming back to approach $q$ ). This is a more important

\footnotetext{
${ }^{6}$ It may be appropriate to mention here that the same is true, even to a higher degree, for Morse theory. There exist tight connections between the indices of the critical points and the diffeomorphism type of $M$, which are best expressed using handle theory. This turns Morse theory into a powerful tool to construct and describe manifolds and places it in a salient position among the differential topologists' resources.
} 

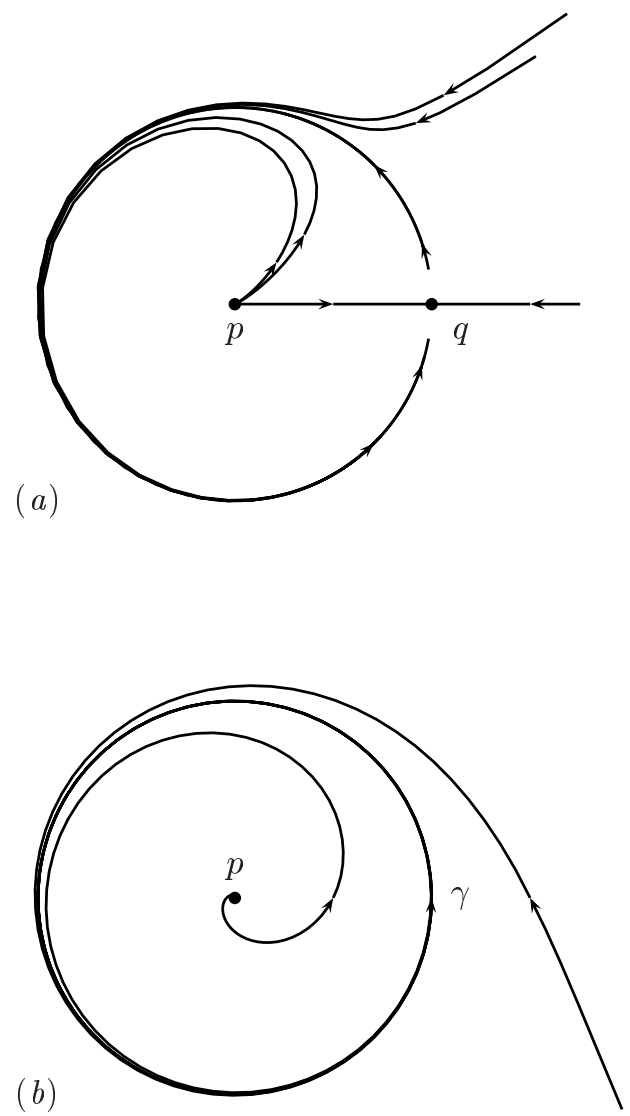

Figure 6. Examples of attractors in the plane.

issue than it may seem at first sight since it cuts down the predictive power of $K$, because it is difficult to decide whether the observed behaviour (for example, numerically computed) of a certain point is transient or representative of its long term evolution. From the mathematical point of view, $K$ lacks the property of stability.

Definition 19 An attractor $K$ is stable if, for every neighbourhood $U$ of $K$, there exists another neighbourhood $V$ such that $p \cdot[0,+\infty) \subseteq U$ for every $p \in V$ (that is, $V \cdot[0,+\infty) \subseteq U$ ). An unstable attractor is an attractor which is not stable.

Definition 19 conveys the idea that trajectories starting close enough to $K$ remain close ever after 7

Example 20 The attractors shown in Figure $6(b)$ and Figure 5 (a) are stable. On the other hand that of Figure 6 (a) is not stable.

Example 21 Let $\mathcal{M}=\left\{M_{1}, \ldots, M_{n}\right\}$ be a Morse decomposition of the compact phase space $M$. Then $M_{1}$ is a stable attractor. Indeed, let $G$ be a Lyapunov function for $\mathcal{M}$ as in Definition 16 and observe that the family $P_{\varepsilon}=G^{-1}\left(\left[c_{1}, c_{1}+\varepsilon\right]\right)$, for $\varepsilon>0$, is a neighbourhood basis of $K_{1}$ comprised of compact, positively invariant sets. Hence if $U$ is any neighbourhood of $K_{1}$ there exists $\varepsilon>0$ such that $P_{\varepsilon} \subseteq U$, and any $p \in P_{\varepsilon}$ satisfies $p \cdot[0,+\infty) \subseteq P_{\varepsilon} \subseteq U$, which proves that $K_{1}$ is stable according to Definition 19

\footnotetext{
${ }^{7}$ The notion of stability appears in many guises in the realm of dynamical systems, and is given different definitions depending on the authors or the particular case being considered. However, there is always a common feeling underlying them, namely that close enough initial conditions will experiment close evolutions when $t \rightarrow+\infty$.
} 
Some sort of converse can be given to Example 21 Indeed,

Proposition 9 If $K$ is a stable attractor, $\alpha(p) \subseteq \partial \mathcal{A}(K)$ for every $p \in \mathcal{A}(K)-K$. Thus if $M$ is compac 8 $\mathcal{M}=\{K, M-\mathcal{A}(K)\}$ is a Morse decomposition of $M$.

Proof. Since $\mathcal{A}(K)$ is an invariant open set, it will be enough to show that $\alpha(p) \cap \mathcal{A}(K)=\emptyset$ for every $p \in \mathcal{A}(K)$. Thus assume that there exists $q \in \alpha(p)$ which is attracted to $K$ and choose a sequence $t_{n} \rightarrow$ $-\infty$ such that $p \cdot t_{n} \rightarrow q$. Given a neighourhood $U$ of $K$, use Definition 19 to find another neighbourhood $V$ of $K$ such that $V \cdot[0,+\infty) \subseteq U$. Observe that $P:=V \cdot[0,+\infty)$ is a positively invariant (by construction) neighbourhood (because it contains $V$ ) of $K$. The hypothesis that $q$ is attracted by $K$ implies that $q \cdot t \in P$ for big enough $t$ and consequently $p \cdot\left(t+t_{n}\right) \in P$ for large $n$. But $t+t_{n} \rightarrow-\infty$, so taking $n$ sufficiently big $t+t_{n}<0$ and $p \in p \cdot\left[t+t_{n},+\infty\right) \subseteq P$. Since $K$ has a arbitrarily small positively invariant neighbourhoods, $p \in K$.

It can be proved (see for example [8]) that for every attractor $K$ there exists a uniquely defined stable attractor $\widehat{K}$, which we shall call the stabilization of $K$, such that $K \subseteq \widehat{K}, \mathcal{A}(K)=\mathcal{A}(\widehat{K})$ and $\widehat{K}$ is the smallest stable attractor with these properties. In fact $\widehat{K}=\{p \in \mathcal{A}(K): \alpha(p) \bigcap K \neq \emptyset\}$, although we shall not prove this.

Stable attractors have been studied from many points of view but a thorough understading of their (possibly very complicated) geometrical properties had to wait until a shape-theoretical approach was taken by Hastings [32]. In our parlance, he proved that the inclusion $j: K \rightarrow P$ in any positively invariant neighbourhood $P \subseteq \mathcal{A}(K)$ of $K$ is a shape equivalence (this is Example 10). Later work by other authors [9], [29], [64] showed that $P$ could be chosen to have the homotopy type of a finite polyhedron. Thus:

Theorem 12 Every stable attractor $K$ in a manifold $M$ has the shape of a finite polyhedron.

By considering shape theory of non-compact spaces (which we shall not present here, but has the same formal properties as SCpt), Kapitanski and Rodnianski [37] proved the following:

Theorem 13 For every stable attractor $K$ the inclusion $j: K \rightarrow \mathcal{A}(K)$ is a shape equivalence.

Theorems 12 and 13 together with the invariance of Čech cohomology under shape equivalences, yield the following

Corollary 5 Let $K$ be a stable attractor in a manifold $M$. Then all its cohomology groups $\check{H}^{k}(K)$ are finitely generated and the inclusion $j: K \rightarrow \mathcal{A}(K)$ induces isomorphisms in cohomology. Furthermore, $\chi(K)$ and $\chi(\mathcal{A}(K))$ are well defined and agree.

Regarding the shape index of a stable attractor, the following result is as complete as one could desire:

Proposition 10 If $K$ is a stable attractor, it is an isolated set and $s(K)=\operatorname{Sh}(K \bigcup *, *)$. Consequently $\chi s(K)=\chi(K)$.

Proof. Let $N$ be any compact neighbourhood of $K$ contained in $\mathcal{A}(K)$. If $p \in N^{-}$, then $\emptyset \neq \alpha(p) \subseteq$ $N \subseteq \mathcal{A}(K)$ (by Example 5) and, by Proposition $9 p \in K$. Thus $N^{-}=K$, so $N$ isolates $K$, and we may as well assume by Theorem 8 that $N$ is an isolating block for $K$. Since $n^{-}=N^{-} \bigcap \partial N=\emptyset$, we get $s(K)=\operatorname{Sh}\left(N^{-} / n^{-}, n^{-} / n^{-}\right)=\operatorname{Sh}(K \bigcup *, *)$. The equality $\chi s(K)=\chi\left(N^{-}, n^{-}\right)=\chi(K)$ is straightforward.

All these results imply that stable attractors are fairly well known from the shape-theoretical point of view (see also [25], [27] and [65] to abound in this observation), and explains the prominent role they play in the realm of dynamical systems. Hence it is clear that deciding whether a given attractor $K$ is stable is of considerable practical interest. Regarding this point, one has for example the following result (see [48] or [61] for more general statements).

\footnotetext{
8 The compactness assumption is included because Morse decompositions are defined for compact phase spaces, but it is otherwise immaterial. Indeed, $M$ (being locally compact) admits a one-point compactification $M_{\infty}$ obtained by adjoining to $M$ the point $\infty$, and the flow $\varphi$ can be extended to a new flow $\varphi_{\infty}$ in $M_{\infty}$ letting $\infty$ be a fixed point. Hence in the general case $\left\{K, M_{\infty}-\mathcal{A}(K)\right\}$ is a Morse decomposition of $M_{\infty}$.
} 
Theorem 14 An isolated fixed point $p \in \mathbb{R}^{2}$ is stable if, and only if, $\chi(\mathcal{A}(p))=1$.

PROOF. Let $\widehat{p}$ be the stabilization of $p$. Clearly the only critical point in $\mathcal{A}(p)$ is $p$, so both $p$ and $\widehat{p}$ are isolated invariant sets which contain the same unique fixed point $p$. Thus by Theorem [10 we have $\chi s(p)=\iota(p)=\chi s(\widehat{p})$, and the latter equals $\chi(\widehat{p})=\chi(\mathcal{A}(p))=1$ by Proposition 10 Theorem 13 and hypothesis. Consequently $\chi s(p)=1$.

Let $p$ be an isolating block for $K$ in the form given by Corollary 4 Then $N$ has the homotopy type of a bouquet of $k$ circumferences, and its exit set is a union of $\ell$ intervals together with some circumferences, so $\chi s(p)=\chi(N)-\chi(L)=1-k-\ell=1$ with $k, \ell \geq 0$. Thus $k=0$ (so $N$ is a disk) and $\ell=0$ (so $L$ is a union of circumferences). If $L$ were nonempty, it would have to be the whole boundary of $N$, which is impossible because then no point could enter $N$ to approach $p$, contradicting the assumption that $p$ is an attractor. Hence $L=\emptyset$ and $N$ is a positively invariant neighbourhood of $p$. Since $N$ can be chosen as small as needed, $p$ is stable.

The boundary $D$ of the region of attraction $\mathcal{A}(K)$ of some attractor $K$ (not necessarily stable, although we are mainly thinking of them here) is a closed invariant set which shows great dynamical interest. Naively speaking, points near $D$ may either approach $K$ asymptotically (if they belong to $\mathcal{A}(K)$ ) or may always remain apart from $K$ (if they do not). These considerations stand at the doorstep of chaos theory and fractal sets, because the latter frequently arise as boundaries of basins of attraction (see for example [54] and references therein). One may also wonder whether certain properties of the attractor $K$ can be extended beyond its region of attraction, and in this context $D$ plays an important role because it is a dynamical barrier between $\mathcal{A}(K)$ and its complementary set in $M$.

In a similar fashion to what happened with attractors, it is natural to study sets like $D$ with shapetheoretical tools, but the picture now is not as easy. Even in apparently simple situations, $D$ can be strongly complicated, as shown in Figure 7

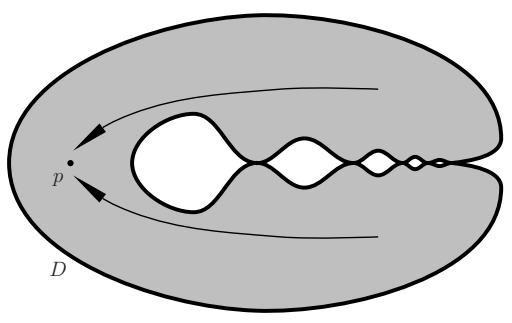

Figure 7. $p$ is a stable attractor, its basin $\mathcal{A}(p)$ is a disk with infinitely many holes.

Still, mild assumptions on the shape index of $K$ provide strong results like the following (see [62]).

Theorem 15 Let $K$ be a stable attractor in a manifold $M$ and assume that the boundary $D$ of its basin of attraction is an isolated set. If $C \breve{H}^{1}(D)=0$, then $D$ has the shape of a finite polyhedron.

Recall that $C \check{H}^{k}(D)$ stands for the $k$-dimensional cohomological index of $K$, which is nothing but the $k$-dimensional Čech cohomology group of the pair $\left(N^{-}, n^{-}\right)$, where $N$ is an isolating block for $D$.

Theorem 16 Let $K$ be a stable attractor in a compac 9 phase space $M$ and assume that the boundary $D$ of its basin of attraction is an isolated set. If $C H^{1}(D)=0$, then there exists a compact invariant set $K^{\prime} \subseteq M$ such that $\mathcal{M}=\left\{K, D, K^{\prime}\right\}$ is a Morse decomposition of $M$.

Moreover, if $M=\mathbb{S}^{n}$ and $K$ has trivial shape, then $\check{H}^{k}\left(K^{\prime}\right)=C H^{n-k-1}(D)$.

A related question to that we have just mentioned regards the properties of the boundary $\partial K$ of a stable attractor $K$, rather than of its basin of attraction. Here one cannot expect the dynamics to be very exciting,

${ }^{9}$ See footnote 8 
but the nature of $\partial K$ may be extremely complicated from the shape-theoretic point of view. This is explored in [63], where the shapes of $\partial K$ and $\partial P$, the latter being a positively invariant compact neighbourhood of the former, are related. For example, one has the following result:

Theorem 17 Let $K$ be a stable attractor in $\mathbb{R}^{n}$ contained in the interior of a positively invariant $n$ ball $\mathbb{D}^{n}$. If $\operatorname{int}(K) \neq \emptyset$, then $\operatorname{Sh}(\partial K) \geq \operatorname{Sh}\left(\mathbb{S}^{n-1}\right)$.

(If $\operatorname{int}(K)$ were empty, then $K=\partial K$ and we know by Example 10 that $\operatorname{Sh}(K)=\operatorname{Sh}\left(\mathbb{D}^{n}\right)=*$ ).

Having dealt with stable attractors so far, it is natural to ask to what extent the results above can be extended to more general classes of attractors. These were first brought into the picture and put on par with stable ones in [4] and [44], at least at the general topology level. Regarding their geometric properties, like those described above in the stable case, not much is known even today. We already mentioned that any attractor $K$ uniquely determines a minimal stable attractor $\widehat{K}$ which contains it and has the same region of attraction as $K$, so one could think that a good approach would be to explore the relation between $K$ and $\widehat{K}$ and then use what we know about stable attractors to deduce things about $K$. However $K$ and $\widehat{K}$ are, so to speak, dynamically entangled. More precisely, the main difficulty here is that they cannot be distinguished by Lyapunov functions (this is basically due to recurrence issues, a generalization of Morse decompositions which may be of interest here is contained in [26]).

Proposition 11 If $K$ is an attractor and $G$ is a Lyapunov function which is constant on $K$, then $G$ is also constant on $\widehat{K}$.

ProOF. We mentioned above that $\widehat{K}=\{p \in \mathcal{A}(K): \alpha(p) \bigcap K \neq \emptyset\}$. Thus if $p \in \widehat{K}$ we have $\alpha(p) \bigcap K \neq \emptyset$ and $\emptyset \neq \omega(p) \subseteq K$ so, since by Proposition 3 the function $G$ is constant on $\alpha(p)$ and $\omega(p)$, it must be the case that $\left.G\right|_{\alpha(p)}=\left.G\right|_{K}=\left.G\right|_{\omega(p)}$. Hence $G$ is constant on $\widehat{K}$.

Thus the Morse theory presented earlier in this paper is useless in this context. Still, some classes of unstable attractors are suitable for a deep examination with topological tools. In particular, K. Athanassopoulos introduced in [2] and [3] the class of attractors with no external explosions, which we shall not define but which encompasses stable attractors and a wide class of unstable ones. Later work on these attractors, prompted by the cited papers, was presented in [48] or [61]. From these let us present the following results.

Theorem 18 Every attractor $K$ with no external explosions in a manifold $M$ has the shape of a finite polyhedron.

Theorem 19 For every attractor $K$ with no external explosions in an even-dimensional manifold $M$, the equality $\chi(K)=\chi(\mathcal{A}(K))$ holds.

Theorem 20 Let $K$ be an unstable attractor with no external explosions in a manifold $M$. If $K$ has the shape of $\mathbb{S}^{2}$, then $M$ is homeomorphic either to $\mathbb{S}^{2} \times \mathbb{S}^{1}$ or to $\mathbb{S}^{2} \times{ }_{t} \mathbb{S}^{1}$, the latter being the result of identifying $\mathbb{S}^{2} \times\{0\}$ and $\mathbb{S}^{2} \times\{1\}$ in $\mathbb{S}^{2} \times[0,1]$ by means of an orientation reversing homeomorphism.

One can easily observe the similarities between these results and the ones presented earlier concerning stable attractors. Theorem 20 in particular, presents a very strong connection between dynamics (the hypothesis that $K$ is an unstable attractor with no external explosions) and global properties of the phase space.

\section{References}

[1] Aarts, J. M., (1988). The structure of orbits in dynamical systems, Fund. Math., 129, 39-57.

[2] Athanassopoulos, K., (2003). Explosions near isolated unstable attractors, Pacific J. Math., 210, 2, $201-214$. 
[3] Athanassopoulos, K., (2006). Remarks on the region of attraction of an isolated invariant set, Colloq. Math., 104, $157-167$.

[4] Auslander, J. and Bhatia, N. P. and Seibert, P., (1964). Attractors in dynamical systems, Bol. Soc. Mat. Mexicana (2), 9, 55-66.

[5] Beck, A., (1958). On invariant sets, Ann. of Math., 67, 1, 99-103.

[6] Bertolim, M. A. and Mello, M. P. and de Rezende, K. A., (2005). Poincaré-Hopf inequalities and Morse inequalities for Lyapunov graphs, Ergodic Theory Dynam. Systems, 25, 1-39.

[7] Bertolim, M. A. and de Rezende, K. A. and Neto, O. M. and Vago, G. M., (2006). Isolating blocks for Morse flows, Geom. Dedicata, 121, 19-41.

[8] Bhatia, N. P. and Szegö, G. P., (1970). Stability theory of Dynamical Systems, Springer-Verlag.

[9] Bogatyй, S. A. and Gutsu, V. I., (1989). On the structure of attracting compacta, Differentsialnye Uravneniya, 25, 5, 907-909, 920 .

[10] Borsuk, K., (1967). Theory of retracts, Państwowe Wydawnictwo Naukowe, 44.

[11] Borsuk, K., (1968). Concerning homotopy properties of compacta, Fund. Math., 62, 223-254.

[12] Borsuk, K., (1975). Theory of Shape, Państwowe Wydawnictwo Naukowe, 59.

[13] Coddington, E. A. and Levinson, N., (1955). Theory of Ordinary Differential Equations, McGraw-Hill.

[14] Conley, C., (1978). Isolated invariant sets and the Morse index, CBMS Regional Conference Series in Mathematics 38

[15] Conley, C. and Easton, R., (1971). Isolated invariant sets and isolating blocks, Trans. Amer. Math. Soc., 158, $35-61$.

[16] Conley, C. and Zehnder, E., (1984). Morse-type Index Theory for Flows and Periodic Solutions for Hamiltonian Equations, Comm. Pure Appl. Math., XXXVII, 207-253.

[17] Churchill, R. C., (1972). Isolated invariant sets in compact metric spaces, J. Diff. Eq., 12, 330-352.

[18] Cruz, R. N. and de Rezende, K. A., (1999). Gradient-like flows on high-dimensional manifolds, Ergodic Theory Dynam. Systems, 2, 2, 339-362.

[19] de Rezende, K. A., (1987). Smale flows on the three-sphere, Trans. Amer. Math. Soc., 303, 1, 283-310.

[20] de Rezende, K. A. and Franzosa, R. D., (1993). Lyapunov graphs and flows on surfaces, Trans. Amer. Math. Soc., 340, 2, 767-784.

[21] Dydak, J. and Segal, J., (1978). Shape theory. An introduction, Lecture Notes in Mathematics 68, Springer.

[22] Floer, A., (1989). Witten's complex and infinite dimensional Morse theory, J. Differential Geometry, 30, $207-222$.

[23] Franks, J., (1985). Nonsingular Smale flows on $\mathbb{S}^{3}$, Topology, 24, 3, 265-282.

[24] Franzosa, R., (1989). The connection matrix for Morse decompositions, Trans. Amer. Math. Soc., 311, 561-592.

[25] Giraldo, A. and Sanjurjo, J. M. R., (1999). On the global structure of invariant regions of flows with asymptotically stable attractors, Math. Z., 232, 4, 739-746.

[26] Giraldo, A. and Sanjurjo, J. M. R., (2007). Singular continuations of attractors, preprint.

[27] Giraldo, A. and Morón, M. A. and Ruiz del Portal, F. R. and Sanjurjo, J. M. R., (2005). Shape of global attractors in topological spaces, Nonlinear Anal., 60, 5, 837-847. 
[28] Guckenheimer, J. and Holmes, P., (1983). Nonlinear oscillations, dynamical systems and bifurcations of vector fiels, Applied Mathematical Sciences 42, Springer-Verlag.

[29] Günther, B. and Segal, J., (1993). Every attractor of a flow on a manifold has the shape of a finite polyhedron, Proc. Amer. Math. Soc., 119, 1, 321-329.

[30] Gutiérrez, C., (1986). Smoothing continuous flows on two-manifolds and recurrences, Ergodic Theory Dynam. Systems, 6, 1, 17-44.

[31] Hartman, P., (1964). Ordinary Differential Equations, John Wiley \& Sons.

[32] Hastings, H. M., (1979). A higher-dimensional Poincaré-Bendixson theorem, Glas. Mat. Ser. III, 14(34), 2, 263268.

[33] Hatcher, A., (2002). Algebraic topology, Cambridge University Press.

[34] Hirsch, M. W., (1976). Differential topology, Graduate Texts in Mathematics, 33, Springer-Verlag.

[35] Hopf, H., (1926). Vektorfelden in n-dimensionalen Mannigfaltigkeiten, Math. Annalen, 96, 225-250.

[36] Hu, S., (1965). Theory of retracts, Wayne State University Press.

[37] Kapitanski, L. and Rodnianski, I., (2000). Shape and Morse Theory of Attractors, Comm. Pure and Appl. Math., LIII, 218-242.

[38] Mardešić, S., (1971). On the shape of the quotient space $S^{n} / A$, Bull. Acad. Polon. Sci. Sr. Sci. Math. Astronom. Phys., 19, 623-629.

[39] Mardešić, S. and Segal, J., (1971). Shapes of compacta and ANR-systems, Fund. Math., 72, 1, 41-59.

[40] Mardešić, S. and Segal, J., (1971). Equivalence of the Borsuk and the ANR-system approach to shapes, Fund. Math., 72, 1, 61-68.

[41] Mardešic̀, S. and Segal, J., (1982). Shape Theory. The Inverse System Approach, North-Holland Publishing Company, 26.

[42] McCord, C. K., (1988). The connection map for attractor-repeller pairs, Trans. Amer. Math. Soc., 308, $195-203$.

[43] McCord, C. K., (1989). On the Hopf index and the Conley index, Trans. Amer. Math. Soc., 313, 2, 853-860.

[44] Mendelson, P., (1960). On unstable attractors, Bol. Soc. Mat. Mexicana (2), 5, 270-276.

[45] Milnor, J., (1963). Morse Theory, Princeton University Press.

[46] Milnor, J., (1965). Topology from the differentiable viewpoint, The University Press of Virginia.

[47] Milnor, J., (1965). Lectures on the h-cobordism theorem. Notes by L. Siebenmann and J. Sondow, Princeton University Press.

[48] Morón, M. A. and Sánchez-Gabites, J. J. and Sanjurjo, J. M. R., (2007). Topology and dynamics of unstable attractors, Fund. Math., (to appear).

[49] Morse, M., (1925). Relations Between the Critical Points of a Real Function of $n$ Independent Variables, Trans. Amer. Math. Soc., 27, 3, 345-396.

[50] Morse, M., (1931). The Critical Points of a Function of $n$ Variables, Trans. Amer. Math. Soc., 33, 1, 72-91.

[51] Mrozek, M., (1990). The Conley index on compact ANRs is of finite type, Results Math., 18, 3-4, $306-313$.

[52] Nemytskii, V. V., (1954). Topological problems of the theory of dynamical systems, Translations of the AMS, 105.

[53] Nemytskii, V. V. and Stepanov, V. V., (1960). Qualitative Theory of Differential Equations, Princeton University Press. 
[54] Nusse, H. E. and Yorke, J. A., (2003). Characterizing the basins with the most entangled boundaries, Ergodic Theory Dynam. Systems, 23, 3, 895-906.

[55] Rybakowski, K. P. (1987). The Homotopy Index and Partial Differential Equations, Springer-Verlag.

[56] Robbin, J. W. and Salamon, D., (1988). Dynamical systems, shape theory and the Conley index, Ergod. Th. \& Dynam. Sys., 8*, 375-393.

[57] Peixoto, M. M., (1962). Structural stability on two-dimensional manifolds, Topology, 1, 101-120.

[58] Poincaré, H., (1881-82, 1885-86) Mémoire sur les courbes définies par une équation différentielle, J. de Math., 7, 375-442 (1881).; 8, 251-296 (1882).; 11, 187-244 (1885).; 12, 151-217 (1886)..

[59] Salamon, D., (1985). Connected simple systems and the Conley index of isolated invariant sets, Trans. Amer. Math. Soc., 291, 1, 1-41.

[60] Salamon, D., (1990). Morse theory, the Conley index, and Floer homology, Bull. London Math. Soc., 22, 113140.

[61] Sánchez-Gabites, J. J., (2007). Unstable attractors in manifolds, (preprint).

[62] Sánchez-Gabites, J. J. and Sanjurjo, J. M. R., (2006). On the topology of the boundary of a basin of attraction, Proc. Amer. Math. Soc., (to appear).

[63] Sánchez-Gabites, J. J. and Sanjurjo, J. M. R., (2007). Shape properties of the boundary of attractors, Glas. Mat., 42(62), 117-130.

[64] Sanjurjo, J. M. R., (1994). Multihomotopy, Čech spaces of loops and shape groups, Proc. London Math. Soc. (3), 69, 2, 330-344.

[65] Sanjurjo, J. M. R., (1995). On the structure of uniform attractors, J. Math. Anal. Appl., 192, 2, 519-528.

[66] Sanjurjo, J. M. R., (2003). Morse equations and unstable manifolds of isolated invariant sets, Nonlinearity, 16, $1435-1448$.

[67] Smale, S., (1960). Morse inequalities for a dynamical system, Bull. Amer. Math. Soc., 66, 43-49.

[68] Smale, S., (1961). On gradient dynamical systems, Ann. of Math., 74, 199-206.

[69] Smale, S., (1963). Stable manifolds for differential equations and diffeomorphisms, Ann. Scuola Norm. Sup. Pisa Ser. 3, 17, 97-117.

[70] Spanier, E. H., (1966). Algebraic Topology, McGraw-Hill.

[71] Wallace, A. H., (1970). Algebraic Topology, W. A. Benjamin Inc.

[72] Ważewski, T., (1947). Sur un principe topologique de l'examen de l'allure asumptotique des intégrales des équations differentiélles ordinaires, Ann. Soc. Polon. Math., 20, 279-313.

[73] Whitney, H., (1933). Regular families of curves, Ann. of Math., 34, 244-270.

[74] Wiggins, S., (1994). Normally hyperbolic invariant manifolds in dynamical systems, Applied Mathematical Sciences 105, Springer-Verlag.

J. J. Sánchez-Gabites

Facultad de Matemáticas,

Departamento de Geometría y Topología,

Universidad Complutense de Madrid.

28084 Madrid, Spain. 